\title{
Article
}

\section{Stellar spectral models compared with empirical data}

Knowles, Adam T, Sansom, Anne E, Coelho, P R T, Prieto, C Allende, Conroy, C and Vazdekis, A

Available at http://clok.uclan.ac.uk/27861/

Knowles, Adam T, Sansom, Anne E ORCID: 0000-0002-2782-7388, Coelho, P R T, Prieto, C Allende, Conroy, C and Vazdekis, A (2019) Stellar spectral models compared with empirical data. Monthly Notices of the Royal Astronomical Society, 486 (2). pp. 1814-1832. ISSN 0035-8711

It is advisable to refer to the publisher's version if you intend to cite from the work. http://dx.doi.org/10.1093/mnras/stz754

For more information about UCLan's research in this area go to http://www.uclan.ac.uk/researchgroups/ and search for <name of research Group>.

For information about Research generally at UCLan please go to http://www.uclan.ac.uk/research/

All outputs in CLoK are protected by Intellectual Property Rights law, including Copyright law. Copyright, IPR and Moral Rights for the works on this site are retained by the individual authors and/or other copyright owners. Terms and conditions for use of this material are defined in the policies page.

\section{CLoK}

Central Lancashire online Knowledge www.clok.uclan.ac.uk

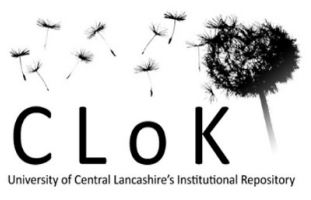




\title{
Stellar spectral models compared with empirical data
}

\author{
Adam T. Knowles ${ }^{\oplus},{ }^{1 \star}$ A. E. Sansom ${ }^{\oplus},{ }^{1}$ P. R. T. Coelho, ${ }^{2}$ C. Allende Prieto, ${ }^{3,4}$ \\ C. Conroy ${ }^{5}$ and A. Vazdekis ${ }^{\oplus 3,4}$ \\ ${ }^{1}$ Jeremiah Horrocks Institute, School of Physical Sciences and Computing, University of Central Lancashire, Preston PRI 2HE, UK \\ ${ }^{2}$ Instituto de Astronomia, Geofísica e Ciêcias Atmosféricas, Univ. de São Paulo, Rua do Matão 1226, 05508-090 São Paulo, Brazil \\ ${ }^{3}$ Instituto de Astrofísica de Canarias, Vía Láctea, E-38205 La Laguna, Tenerife, Spain \\ ${ }^{4}$ Universidad de La Laguna, Departamento de Astrofísica, E-38206 La Laguna, Tenerife, Spain \\ ${ }^{5}$ Department of Astronomy, Harvard University, Cambridge, MA 02138, USA
}

Accepted 2019 March 1. Received 2019 February 1; in original form 2018 May 25

\begin{abstract}
The empirical MILES stellar library is used to test the accuracy of three different, state-of-theart, theoretical model libraries of stellar spectra. These models are widely used in the literature for stellar population analysis. A differential approach is used so that responses to elemental abundance changes are tested rather than absolute levels of the theoretical spectra. First we directly compare model line strengths and spectra to empirical data to investigate trends. Then we test how well line strengths match when element response functions are used to account for changes in $[\alpha / \mathrm{Fe}]$ abundances. The aim is to find out where models best represent real star spectra, in a differential way, and hence identify good choices of models to use in stellar population analysis involving abundance patterns. We find that most spectral line strengths are well represented by these models, particularly iron- and sodium-sensitive indices. Exceptions include the higher order Balmer lines $(\mathrm{H} \delta, \mathrm{H} \gamma)$, in which the models show more variation than the data, particularly at low temperatures. $\mathrm{C}_{2} 4668$ is systematically underestimated by the models compared to observations. We find that differences between these models are generally less significant than the ways in which models vary from the data. Corrections to $\mathrm{C}_{2}$ line lists for one set of models are identified, improving them for future use.
\end{abstract}

Key words: techniques: spectroscopic-stars: abundances - stars: atmospheres.

\section{INTRODUCTION}

Element abundance patterns in galaxies are well known to contain information about the formation history of their constituent stellar populations (e.g. Worthey, Faber \& Gonzalez 1992; Proctor \& Sansom 2002; Trager et al. 2000; Thomas et al. 2005). Even medium-resolution spectra of galaxies contain detailed information regarding abundance patterns. The dominant sources of interstellar medium (ISM) enrichment are Type II and Type Ia supernovae. Supernovae from massive star progenitors enrich the ISM with a range of heavy elements over time-scales of less than $10^{8} \mathrm{yr}$. Type Ia supernovae, from white dwarf progenitors, enrich the ISM with mainly iron-peak elements over longer time-scales, ranging from prompt explosions of $\approx 10^{8} \mathrm{yr}$ to a more delayed enrichment of up to $\approx 10^{10} \mathrm{yr}$ (Sullivan et al. 2006; Mannucci 2008; Pagel 2009; Maoz, Sharon \& Gal-Yam 2010). The time-scales of elemental production in the two types of supernovae are different; therefore, it is possible to use the ratio of $\alpha$-capture and iron-peak elements (e.g. from

*E-mail: atknowles@uclan.ac.uk observations of $[\mathrm{Mg} / \mathrm{Fe}]^{1}$ ) as a clock to constrain the time-scale over which the stars were born. Abundance patterns in galaxies can be measured using spectral indices or full spectrum fitting. We describe these two approaches below.

One way to measure abundance ratios from observing integrated populations is to measure spectral indices and compare with model values. Commonly, such indices are defined by three bandpasses, a feature band and two sidebands (pseudocontinua), and are then measured as a pseudo-equivalent width. The most popular system of indices is the LICK/IDS system (e.g. Worthey 1994; Worthey \& Ottaviani 1997) that defines 25 spectral indices between 4000 and $6500 \AA$, although other systems have been designed for use with spectral libraries at different spectral resolutions, e.g. the Line Index System (LIS) MILES system (Vazdekis et al. 2010). There are other ways to define indices, such as flux ratio indices (Rose 1984) or indices based on the D4000 feature (Poggianti \& Barbaro 1997). With an index system defined, it can be used to investigate the properties of stellar populations in galaxies. Moreover, it is possible

\footnotetext{
${ }^{1}[\mathrm{~A} / \mathrm{B}]=\log [n(A) / n(B)]_{*}-\log [n(A) / n(B)]_{\odot}$, where $n(A) / n(B)$ is the number
} abundance ratio of element $\mathrm{A}$, relative to element $\mathrm{B}$. 
to study how the indices respond to elemental abundance changes using theoretically produced stellar spectra. The results are usually presented in the form of response functions, which are tables that show how spectral features are affected by abundance changes. This type of study was first performed in the work of Tripicco \& Bell (1995) with the assessment of 10 elements using synthetic spectra. A developed version of this study, whose derived response functions have been widely used to date, was then carried out by Korn, Maraston \& Thomas (2005). They used updated linelists and atomic transition probabilities with more accurate atmospheric models and also incorporated a range of metallicities. Sansom et al. (2013) tested the differential behaviour of the Korn et al. (2005) models, via response functions and found deviations in the higher order Balmer features between those models and empirical star data. More updated and larger numbers of theoretical spectra have been used in more recent studies (e.g. Lee et al. 2009; Holtzman et al. 2015). With measures of how spectral indices are sensitive to elemental abundances, one can use the derived response functions to differentially correct indices to account for changes in abundance patterns. There are many applications of such work throughout the literature in both Milky Way and extragalactic studies (e.g. Trager et al. 2000; Proctor \& Sansom 2002; Schiavon 2007; Thomas, Johansson \& Maraston 2011; Onodera et al. 2015; Sesto et al. 2018).

Another approach to account for different abundance patterns is full spectrum fitting. Some of the first work to take a differential abundance pattern approach in full spectrum fitting of stellar populations was that of Prugniel et al. (2007) followed by that of Walcher et al. (2009), for the modelling of $\alpha$-enhanced Simple Stellar Populations (SSPs). This work was then expanded by Conroy \& van Dokkum (2012), by varying 11 elements separately. Vazdekis et al. (2015) performed a similar approach to Walcher et al. (2009), focusing on an $\alpha$ enhancement in SSPs. A similar method for differentially correcting individual star spectra can be used to account for variations in abundance patterns. If accurate measures are made that quantify how spectral indices or full spectra respond to elemental abundances, it is possible to begin to build stellar spectral libraries that contain abundance patterns different from our own solar neighborhood. Such libraries allow one to produce stellar population synthesis models that include stars with abundance patterns that differ from the Milky Way. This is motivated by the different abundance patterns seen in giant Early-Type and Dwarf Spheroidal galaxies (e.g. Letarte, Hill \& Tolstoy 2007; Conroy, Graves \& van Dokkum 2014).

Differentially correcting empirical stellar spectra relies on the accuracy of the theoretical stellar spectra used. With a large number of models currently available, each with their own set of advantages, assumptions, and limitations, deciding which synthetic spectra to use is difficult. Here we test the predictions of three stellar spectral model libraries against empirical star data in the context of abundance patterns, with the aim of highlighting current strengths and weaknesses of the models. These models represent some of the most recent works in stellar population analysis, covering a broad range of parameter space, suitable for modelling integrated stellar populations. This work expands on Sansom et al. (2013), testing more state-of-the-art theoretical stellar spectral models.

The structure for this paper is as follows. Section 2 describes the three models of stellar spectra that are tested in this study. Section 3 outlines the MILES empirical spectra used in the comparison. In Section 4 we directly compare Lick indices of MILES stars to those predicted from theoretical stellar spectra. Section 5 presents a differential approach, using response functions, in which we compare normalized Lick indices from empirical MILES stars to those predicted from theoretical response functions. Section 6 discusses the findings and possible physical reasons for model disagreements, through analysis of both indices and full spectra. Section 7 presents our conclusions.

\section{MODELS OF STELLAR SPECTRA}

Throughout this paper we will be using three model libraries of stellar spectra, produced by three independent authors to test responses of the models to changes in abundance pattern, relative to solar. The models we have chosen are state-of-the-art in the context of stellar population analyses from integrated light. They have been created for use in stellar population modelling, covering a wide range of stellar parameters and abundance patterns. Some recent works applying these models can be found in Conroy et al. (2014), Holtzman et al. (2015), and Vazdekis et al. (2015). These models built on the first predictions of SSP spectra with abundance variations from the works of Coelho et al. (2007), Prugniel et al. (2007), Percival et al. (2009), and Lee et al. (2009). All of these works predict the spectra of stellar populations with abundance variations, rather than the classical approach of predicting indices. This section describes and outlines the codes and parameters used in the production of the theoretical stellar spectra from each of three modellers.

Generation of synthetic spectra requires two main steps. First, calculation of the model atmosphere provides a mathematical model describing the variation of physical parameters such as density, temperature, and pressure as a function of radial depth, for an assumed star type and composition. The second step is to pass photons through the generated atmosphere to compute an emergent spectrum. This requires the use of a synthetic spectrum code together with a list of line and molecular absorption transitions and a specification of element abundances. The self-consistent approach to generate a theoretical stellar spectrum would be to exactly match the abundances in both steps of the production. To reduce computational time, a simplification is made in which only the dominant sources of opacity are varied in the model atmosphere whilst more elements are varied in the synthetic spectrum. However if one uses ATLAS12 (Kurucz 1996) or OMARCS (Gustafsson et al. 2008) model atmosphere codes, it is possible to have the same abundance pattern in both components of the spectrum generation.

One of the most commonly used codes to generate model atmospheres is ATLAS (Kurucz 1979 and updates), a one-dimensional, local thermodynamic equilibrium and plane-parallel code. The original code provides a base on which developments have been made, e.g. ATLAS9 (Kurucz 1993) and ATLAS12 (Kurucz 2005; Castelli 2005a). An important effect in the generation of stellar photospheres is the line opacity due to atomic (and molecular) line absorption. Line opacity depends on temperature, pressure, chemical composition, and microturbulence $\left(v_{\text {turb }}\right)$. Statistical methods were developed to deal with the vast number of lines present in stellar atmospheres. The method implemented provides one of the biggest differences between the versions of the ATLAS code. ATLAS 9 uses Opacity Distribution Functions (ODFs) as an approach to this problem. ODFs treat the line opacities in a given frequency interval by a smoothly varying function. The ODFs have to be computed for a particular abundance pattern before generating the model atmospheres. ATLAS12 uses the Opacity Sampling method (OS) to compute the line opacity at a number of frequency points.

Another important parameter in the computation of stellar spectra

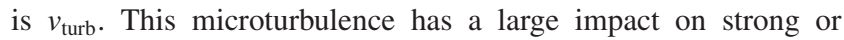


saturated lines, and therefore the choice of this parameter when calculating a synthetic spectrum will affect the resulting line strengths. In order to gain an understanding of line-strength uncertainties involved with the $v_{\text {turb }}$ parameter, we produced several models changing $v_{\text {turb }}$ (see Section 4).

In this paper we test three star types with varying element abundances that represent Cool Dwarf (CD) $\left(T_{\text {eff }}=4575 \mathrm{~K}, \log \right.$ $g=4.60 \mathrm{dex})$, Cool Giant (CG) $\left(T_{\mathrm{eff}}=4255 \mathrm{~K}, \log g=1.90 \mathrm{dex}\right)$, and Turn-off (TO) $\left(T_{\text {eff }}=6200 \mathrm{~K}, \log g=4.10\right)$ stars with the same parameters as in Korn et al. (2005) and the analysis of Sansom et al. (2013). These star types are chosen as they are representative of stars present in older stellar populations that future work will focus on, using results from this study.

Below we specify the codes used by the three modellers to produce spectra, the wavelength range, sampling, elements varied, and stellar parameters used. Table 1 summarizes this information. All models assume that the $\alpha$-capture group elements are $\mathrm{O}, \mathrm{Ne}$, $\mathrm{Mg}$, Si, Ca, and Ti, unless otherwise stated. Spectra with abundance patterns of Solar and those in Table 2 were provided. Table 2 summarizes the $T_{\text {eff }}, \log g$, and element enhancements provided by each modeller, for use in Section 5 . The $[\mathrm{M} / \mathrm{H}]$ value in Table 2 is defined as a scaled metallicity.

\subsection{Conroy}

Theoretical star spectra from Conroy were made using the ATLAS12 atmosphere code and SYNTHE (Kurucz \& Avrett 1981) spectral synthesis package. Groups of Cool Dwarf, Cool Giant, and Turn-off star spectra were made with a wavelength range of 3700-10000 and sampling of $\Delta \log \lambda(\AA)=2.17 \times 10^{-5}$. It is worth highlighting here that only spectra with a $\mathrm{C}+0.15$ dex variation (compared with $\mathrm{C}+0.3$ dex of the other two authors) were provided, which will impact on the derived responses for indices that are particularly sensitive to carbon abundances. The reason for this was to avoid the generation of carbon stars. The solar abundances adopted in the model atmosphere and synthetic spectrum code were from Asplund et al. (2009). Note that the stellar parameters used in producing the model atmospheres were slightly different from the parameters of the other two modellers. This was because these models already existed prior to the current work, rather than being created specifically for this project (as in the other two cases). Further description of the stellar spectral models can be found in Conroy \& van Dokkum (2012). Please note that the native resolution and wavelength range of the models presented in Conroy \& van Dokkum (2012) is higher than that given in Table 1, but the spectra were downsampled and cut at $10000 \AA$. The line lists used in the production of Conroy's models are described in Conroy \& van Dokkum (2012) and are based on lists compiled by Kurucz. ${ }^{2}$ Some of the differences between the three models seen in Sections 4 and 5 may be explained by the inclusion of predicted lines (PLs) in Conroy models that are not present in the other two model libraries. The PLs were included in the Conroy models provided because there were generated for other applications, particularly to compute broad-band colours, which are known to be underestimated if PLs are missing (e.g. section 3 of Coelho 2014 and section 3.2 of Coelho et al. 2007). Most of the PLs are weak, and therefore contribute to the overall continuum shape. However, there are cases of strong PLs that produce lines that disagree with observations, particularly in the bluer parts of the spectrum (see bottom panel of figs 2 and

${ }^{2}$ http://kurucz.harvard.edu
3 of Munari et al. 2005 as well as figs 7-18 of Bell, Paltoglou \& Tripicco 1994). The PLs affect the absolute comparisons more than the differential comparisons.

\subsection{Coelho}

Theoretical star spectra provided by Coelho used both the ATLAS12 model atmosphere code and SYNTHE (Kurucz \& Avrett 1981; Sbordone et al. 2004) spectral synthesis code to generate groups of spectra for a Cool Dwarf, Cool Giant, and Turn-off star. The original wavelength range of the spectra was $3000-8005 \AA$ with a sampling of $\Delta \log \lambda(\AA)=1.4 \times 10^{-6}$. The solar abundances used in the model atmosphere and synthetic spectrum code were those of Grevesse \& Sauval (1998). These models are different from those previously published by Coelho, which were based on ATLAS9 (Coelho 2014). The atomic line lists used in Coelho's models are a combination of lists from Coelho et al. (2005), Castelli (2005a), and Castelli (2005b). In this work we adopt the same molecular opacities as in Coelho (2014), with the following updates ${ }^{3}: C_{2}$ D-A (from Brooke et al. 2013), CH (from Johnson et al. 2014, with energy levels substituted from Zachwieja 1995; Zachwieja 1997; Colin \& Bernath 2010; Bembenek, Kepa \& Rytel 1997; Kepa et al. 1996), and CN A-X and B-X (from Brooke et al. 2014). During the progress of this work, we identified that the file regarding the transition D-A of the molecule $\mathrm{C}_{2}$ used in Coelho (2014) was corrupted. We therefore warn that the predictions of that library around the main $\mathrm{C}_{2}$ features should be taken with care. This is illustrated in Appendix A, where we compare the corrupted and corrected models. This corruption is likely to be the origin of the strong missing opacity around 4000 $\AA$ in the second panel of fig. 10 in Coelho (2014), which can be attributed to Swan Bands. Note that this problem did not affect earlier models, including Coelho et al. (2005), Coelho et al. (2007), or Vazdekis et al. (2015).

\subsection{Allende prieto}

The spectra provided by Allende Prieto (hereafter referred to as CAP) were made using the ATLAS9 model atmosphere code along with the ASS $\epsilon$ T (Koesterke 2009) spectral synthesis software, used in 1-D. The wavelength range of the spectra was 1200-30000 with a sampling of $\Delta \log \lambda(\AA)=6.5 \times 10^{-7}$. The solar abundance used in both the model atmosphere and synthetic spectrum code was that of Asplund et al. (2005). Further details of the models can be found in Allende Prieto et al. (2014). The line lists used in the CAP models are detailed in Mészáros et al. (2012) and are based on Kurucz lists.

For the CAP models that we generate for Section 4, we use ATLAS9. We direct interested readers to Mészáros et al. (2012) and web pages for the ATLAS-APOGEE survey analysis ${ }^{4}$ for further information on the ODFs and models used in this analysis. Currently for PL9, the ODFs publicly available from the ATLAS-APOGEE website provide a range of abundances in $[\mathrm{M} / \mathrm{H}],[\alpha / \mathrm{M}]$, and $[\mathrm{C} / \mathrm{M}]$. $[\mathrm{M} / \mathrm{H}]$ here is defined as a scaled metallicity. This definition means elements with $Z>2$ are all scaled together e.g. $[\mathrm{M} / \mathrm{H}]=0.2$ means $[\mathrm{Fe} / \mathrm{H}]=0.2=[\mathrm{X} / \mathrm{H}]$, where $\mathrm{X}=3,4, \ldots, 99$. Note that with these definitions, $[\mathrm{M} / \mathrm{H}]$ represents all elements other than the $\alpha$-capture

\footnotetext{
${ }^{3}$ As made available by R. Kurucz; downloaded on 2016 December from http://kurucz.harvard.edu/molecules.html

${ }^{4}$ http://www.iac.es/proyecto/ATLAS-APOGEE/
} 
Table 1. Codes and parameters used by the three modellers when generating their theoretical spectra. These parameters are for spectra used in the derivation of response functions in Section 5. In the final column we specify the consistency of abundance specification between the model atmosphere (MA) generation and radiative transfer $(\mathrm{RT})$ process.

\begin{tabular}{|c|c|c|c|c|c|c|c|}
\hline Model & $\begin{array}{l}\text { Atmosphere } \\
\text { code }\end{array}$ & $\begin{array}{l}\text { Synthetic } \\
\text { spectrum code }\end{array}$ & $\begin{array}{l}\text { Wavelength } \\
\text { range }(\AA)\end{array}$ & $\begin{array}{l}\text { Sampling } \\
(\Delta \log \lambda(\AA))\end{array}$ & $v_{\text {turb }}(\mathrm{km} / \mathrm{s})$ & Solar abundance reference & $\begin{array}{l}\mathrm{MA}+\mathrm{RT} \\
\text { compatible }\end{array}$ \\
\hline Conroy & ATLAS12 & SYNTHE & $3700-10000$ & $2.17 \times 10^{-5}$ & 2 & Asplund et al. (2009) & Yes \\
\hline $\begin{array}{l}\text { Allende Prieto } \\
\text { (CAP) }\end{array}$ & ATLAS9 & $\mathrm{ASS} \in \mathrm{T}$ & $1200-30000$ & $6 \times 10^{-7}$ & 1.5 & $\begin{array}{l}\text { Asplund, Grevesse \& } \\
\text { Sauval (2005) }\end{array}$ & Yes $(\mathrm{C}, \mathrm{M}, \alpha)$ \\
\hline
\end{tabular}

Table 2. $T_{\text {eff }}, \log g$, and element enhancements above solar ( 0.3 dex unless stated otherwise) for the three star types, provided by the modellers for the response function analysis in Section 5. The $[\mathrm{M} / \mathrm{H}]$ column in this table is for the specific case of all metals increased by 0.3 dex.

\begin{tabular}{|c|c|c|c|c|c|c|c|c|c|c|c|c|c|}
\hline Model & $\mathrm{T}_{\text {eff }}(\mathrm{CD}, \mathrm{CG}, \mathrm{TO})(\mathrm{K})$ & $\log \mathrm{g}(\mathrm{CD}, \mathrm{CG}, \mathrm{TO})(\mathrm{dex})$ & $\mathrm{C}$ & $\mathrm{N}$ & $\mathrm{O}$ & $\mathrm{Mg}$ & $\mathrm{Fe}$ & $\mathrm{Ca}$ & $\mathrm{Na}$ & $\mathrm{Si}$ & $\mathrm{Cr}$ & $\mathrm{Ti}$ & {$[\mathrm{M} / \mathrm{H}]$} \\
\hline CAP & $4575,4255,6200$ & $4.60,1.90,4.10$ & $\checkmark$ & $\checkmark$ & $\checkmark$ & $\checkmark$ & $\checkmark$ & $\checkmark$ & $\checkmark$ & $\checkmark$ & $\checkmark$ & $\checkmark$ & $\checkmark$ \\
\hline
\end{tabular}

elements if there is an $\alpha$ enhancement or deficiency (e.g. if $[\mathrm{M} / \mathrm{H}]=$ 0.2 and $[\alpha / \mathrm{M}]=0.1$, this means that $[\alpha / \mathrm{H}]=0.3$ and $[\mathrm{Fe} / \mathrm{H}]=0.2$ ).

\section{EMPIRICAL STELLAR SPECTRA}

The empirical data are from the Medium resolution Isaac Newton Library of Empirical Spectra (MILES) (Sánchez-Blázquez et al. 2006). Whilst stars from our Galaxy do not cover the full parameter range of stars in other galaxies, they do cover a broad range in stellar parameters. These empirical spectra have a wavelength range of 3500-7500 $\AA$, resolution (FWHM) of $2.5 \AA$, and sampling of $0.9 \AA$ (Falcón-Barroso et al. 2011). They have a typical signal-tonoise ratio of over $100 \AA^{-1}$, apart from stars that are members of globular clusters. Of the 985 stars in MILES, Milone, Sansom \& Sánchez-Blázquez (2011) measured the $[\mathrm{Mg} / \mathrm{Fe}]$ abundances for 752 stars. We use their $[\mathrm{Mg} / \mathrm{Fe}]$ measurement as a proxy for all $[\alpha / \mathrm{Fe}]$ abundances in these stars. Therefore MILES is a stellar library for which we know attributes of effective temperature $\left(T_{\text {eff }}\right)$, surface gravity $(\log g)$, metallicity $([\mathrm{Fe} / \mathrm{H}])$, and abundance ratios $([\alpha / \mathrm{Fe}])$ for a large proportion of the whole library. This, with the MILES spectra, allows us a uniformly calibrated data set of stars to test theoretical spectra. We initially use a sub-sample of 51 of the 752 stars that matched the $T_{\text {eff }}$ and $\log g$ parameters of the three theoretical stars described in Section 2, within the observational errors. Stars were chosen that were within $\Delta T_{\text {eff }} \leq \pm 100 \mathrm{~K}, \Delta \log$ $\mathrm{g} \leq \pm 0.2$ ) of the Cenarro et al. (2007) atmospheric parameters, for three specific star types. These limits led to a sample of $7 \mathrm{Cool}$ Dwarfs, 13 Cool Giants, and 31 Turn-off stars (see Sansom et al. 2013, table A1 for details of these individual star parameters and Lick indices). Therefore we have both MILES spectra and their Lick indices available for testing. Whilst full spectrum fitting has become increasingly popular for stellar population analysis in recent years, Lick indices are useful for testing properties of theoretical spectra against observations because they focus on the strongest spectral features. We use the $T_{\text {eff }}, \log g,[\mathrm{Fe} / \mathrm{H}]$, and $[\mathrm{Mg} / \mathrm{Fe}]$ values of the MILES stars presented in table A1 of Sansom et al. (2013), based on parameters in Cenarro et al. (2007), unless stated otherwise. The errors on the measured MILES Lick indices were computed by Sánchez-Blázquez (private communication), from the error spectra obtained by propagating uncertainties throughout the reductions, including flux and wavelength calibration, as well as the errors in the velocity calculations, for each star.

\section{DIRECT COMPARISONS}

The first test we perform directly compares MILES and theoretical star Lick indices. New models are generated that match the MILES stars exactly in $T_{\text {eff }}, \log g,[\mathrm{Fe} / \mathrm{H}]$, and $[\alpha / \mathrm{Fe}]$ for Coelho and CAP models. The theoretical spectra were degraded to the MILES resolution of FWHM = 2.5 $\AA$ (Falcón-Barroso et al. 2011) using a convolution code produced in PYTHON and then resampled to match existing MILES sampling of $0.9 \AA$. Indices are then measured for both the MILES stars and corresponding theoretical star using LECTOR software (Vazdekis 2011). This approach of directly producing models was made for both the Coelho and CAP models, to compare to the sub-sample of 51 MILES stars, described in Section 3. Rather than generating models directly for this comparison, spectra were created for Conroy models using an interpolation within a preexisting grid presented in Conroy \& van Dokkum (2012). Four of the 51 MILES stars fell outside of the parameter range in the grid and were therefore not modelled for Conroy in this comparison. The missing stars were three Turn-off stars (HD084937, HD338529, and $\mathrm{BD}+092190)$ and one cool giant star (HD131430). Although this direct comparison will assess the absolute behaviour of models, the main purpose of this test is to look for trends between models rather than absolute agreement between models and empirical data. The absolute test we perform here will aid the assessment of the differential test performed in Section 5.

The available measured MILES star parameters are $T_{\text {eff }}, \log g$, $[\mathrm{Fe} / \mathrm{H}]$, and $[\mathrm{Mg} / \mathrm{Fe}]$. CAP models are generated by specifying $T_{\text {eff }}$, $\log g,[\mathrm{M} / \mathrm{H}],[\alpha / \mathrm{M}]$, and $[\mathrm{C} / \mathrm{M}]$ and $v_{\text {turb }}$. Therefore, conversions from MILES parameters to the model parameters are required, in addition to assumptions of $[\mathrm{C} / \mathrm{M}]$ and $v_{\text {turb }}$ for the empirical stars. The choice of $v_{\text {turb }}$ to use in the models is explained in Section 4.1 and the conversion process is described in Section 4.2.

\subsection{Microturbulent velocity}

To investigate the effects of microturbulent velocity on the differential application of theoretical line-strengths, three different star 
models were produced using the codes of Allende Prieto et al. (2014). For each base star type (Cool Dwarf, Cool Giant, and Turnoff), we produced an $[\alpha / \mathrm{M}]=0.25 \mathrm{dex}$ and $\mathrm{a}[\alpha / \mathrm{M}]=0$ dex spectrum at $v_{\text {turb }}=1 \mathrm{~km} \mathrm{~s}^{-1}\left(v_{1}\right)$ and $2 \mathrm{~km} \mathrm{~s}^{-1}\left(v_{2}\right)$. All the models were produced with the same sampling of $\Delta \log \lambda(\AA)=0.025 \AA$ (at 3000 $\AA$ ) to isolate the effects of $v_{\text {turb }}$. The models were blurred to MILES FWHM of $2.5 \AA$ and resampled to MILES linear sampling of 0.9 $\AA$ using the same procedure as described previously. LECTOR was then used to compute the line strengths. To assess the differential effect, we took a difference of line strengths through:

$$
\begin{aligned}
& \left(v_{2}\left(\left[\frac{\alpha}{M}\right]=0.25\right)-v_{2}(\odot)\right) \\
& \quad-\left(v_{1}\left(\left[\frac{\alpha}{M}\right]=0.25\right)-v_{1}(\odot)\right),
\end{aligned}
$$

where $v_{i}$ represents the spectrum with $v_{\text {turb }}=i \mathrm{~km} \mathrm{~s}^{-1}$ and indices are measured from these model spectra. Table 3 shows the linestrength indices measured for each of the models as well as the differential effects.

In general, the effect of $v_{\text {turb }}$ on the Cool Dwarf line strengths is smallest, with typical differences of $0.2 \AA$ between 1 and $2 \mathrm{~km} \mathrm{~s}^{-1}$, respectively. The microturbluent velocity has a far greater effect on the Cool Giant spectra with several features differing by order $\sim$ 1-2 $\AA$, particularly $\mathrm{H} \gamma_{A}, \mathrm{G} 4300$, and Fe5015, with a change in $v_{\text {turb }}$ from $1 \mathrm{~km} \mathrm{~s}^{-1}$ to $2 \mathrm{~km} \mathrm{~s}^{-1}$. The Turn-off stars are also significantly affected by $v_{\text {turb }}$. For all star types the differential $v_{\text {turb }}$ effect is small; as can be seen in the $v_{2}(\delta)-v_{1}(\delta)$ of Table 3 . Our findings show that these differences are generally much smaller $(\sim 0.02 \mathrm{dex}$; see Table 3.) than the observational errors on line strengths $(\sim 0.1$ dex; see table 2 of Sansom et al. 2013)

For simplicity, we have chosen to use a constant value of $v_{\text {turb }}=1.5 \mathrm{kms}^{-1}$ for all our models used in this paper, unless otherwise stated. This choice is motivated by larger studies of stars in our Galaxy, where $v_{\text {turb }}$ is measured between the 1 and $2 \mathrm{kms}^{-1}$ (e.g. Holtzman et al. 2015).

\subsection{Abundances in CAP models}

Two approximations are made in the format conversion process for element abundances. First, it is assumed that $[\mathrm{Mg} / \mathrm{Fe}]$ is a proxy for $[\alpha / \mathrm{Fe}]$. This is a reasonable assumption for solar neighbourhood stars, like the 51 MILES stars used in this study, as is shown by the work of Delgado Mena et al. (2010) and Holtzman et al. (2015). A second approximation of $[\mathrm{C} / \mathrm{Fe}]=0$ for the MILES stars was made based on results from da Silva, Milone \& Reddy (2011) and Holtzman et al. (2015) for stars in our Galaxy.

The $[\mathrm{Mg} / \mathrm{Fe}]$ and $[\mathrm{Fe} / \mathrm{H}]$ abundances of the MILES stars are matched in the generated CAP model through $[\alpha / \mathrm{M}]$ and $[\mathrm{M} / \mathrm{H}]$, respectively. Therefore we use the assumption that $[\mathrm{Fe} / \mathrm{H}] \approx[\mathrm{M} / \mathrm{H}]$ and $[\mathrm{Mg} / \mathrm{Fe}] \approx[\alpha / \mathrm{M}]$. [C/Fe] values of the MILES stars are assumed to be 0 throughout, meaning that $[\mathrm{C} / \mathrm{M}]=0$ in the generated models. For these CAP models, solar abundances are defined on the Asplund et al. (2005). Using these conversions, spectra are generated in a selfconsistent way, with the abundances of $\alpha$ and $\mathrm{C}$ varied in the same way for both model atmosphere and spectral synthesis calculations.

\subsection{Absolute comparisons}

Figs 1-5 show direct comparisons between the measured MILES Lick indices and corresponding model Lick indices for these MILES matched spectra.
Fig. 1 shows that the absolute line strengths of the higher order Balmer lines and $\mathrm{H} \beta$ for Conroy, Coelho, and CAP models deviate from observations and this effect increases towards more negative line strengths and cooler temperatures. Fig. 2 shows that Conroy, Coelho, and CAP models predict iron-sensitive features qualitatively well in the absolute comparison, with no strong systematic deviations from the 1:1 agreement lines. Fig. 3 shows a good agreement, over a broad range in index strengths, particularly for CAP models compared with observations for magnesiumsensitive features. There are slight overpredictions of line strengths for Coelho and CAP Cool Giant models, whilst the Conroy cool star models overpredict these magnesium features the most, with clear systematic offsets. Fig. 4 shows that Conroy, Coelho, and CAP all underpredict the line strength indices in $\mathrm{C}_{2} 4668$ and show less variation than is present in MILES stars. Moreover, Conroy and CAP models overpredict the line strength indices of $\mathrm{CN}_{1}$ and $\mathrm{CN}_{2}$ for the cool stars. Fig. 5 shows the absolute predictions of the Conroy, Coelho, and CAP models for calcium- and sodium-sensitive indices agree well with the observations. However, differences between models can be seen in the Ca4455 index, with Coelho Cool Giant models having a tighter relation to the 1:1 line and Conroy models showing systematic overpredictions of this line strength. For Ca4227, the scatter is larger for the cool stars, with all three models behaving similarly. Despite the differences seen between models in Figs 1-5, it is interesting to note how similar the three set of models behave in general, given the different approaches, inputs, and codes of the three models. This tells us that the models are producing similar predictions of the physical processes, although there are still large differences between models and observations in absolute terms.

\section{RESPONSE FUNCTIONS AND THEIR APPLICATION}

The results of Section 4 highlight the disagreements between the models and MILES stars in absolute terms. Other studies have also shown wavelength-dependent disagreements between theoretical models and observed spectra (e.g. Martins \& Coelho 2007; Bertone et al. 2008; Coelho 2014; Villaume et al. 2017; Allende Prieto et al. 2018). One method to incorporate both the abundance pattern predictions provided by theoretical models and the reliability of empirical libraries is to use theoretical spectra to differentially correct empirical spectra. Variations to Lick indices, due to changes in stellar atmospheric abundances, can be quantified in terms of response functions (Tripicco \& Bell 1995). These can be applied to change empirical or theoretical line-strengths due to variations in abundance patterns, particularly differences from solar neighbourhood abundances. We produce response function tables for the models of three star types: a Cool Dwarf, Cool Giant, and a Turn-off star, described in Section 2. To test the responses of different theoretical models to abundance pattern changes, we compare their normalized Lick indices predictions to measured Lick indices of existing MILES stars (described in Section 3).

We test the response functions, derived from theoretical spectra of the three star types, by applying them to a theoretical solar abundance pattern (base) star to account for changes in abundance patterns, namely $[\alpha / \mathrm{Fe}]$ changes. The base model star has the same atmospheric parameters of $T_{\text {eff }}$ and $\log g$ as a chosen MILES base star, within observational errors. The response functions will be used to modify Lick indices of the base model star to account for an abundance pattern of an existing MILES star with same $T_{\text {eff }}$ and $\log g$ as the base star, referred to as an enhanced star. This 


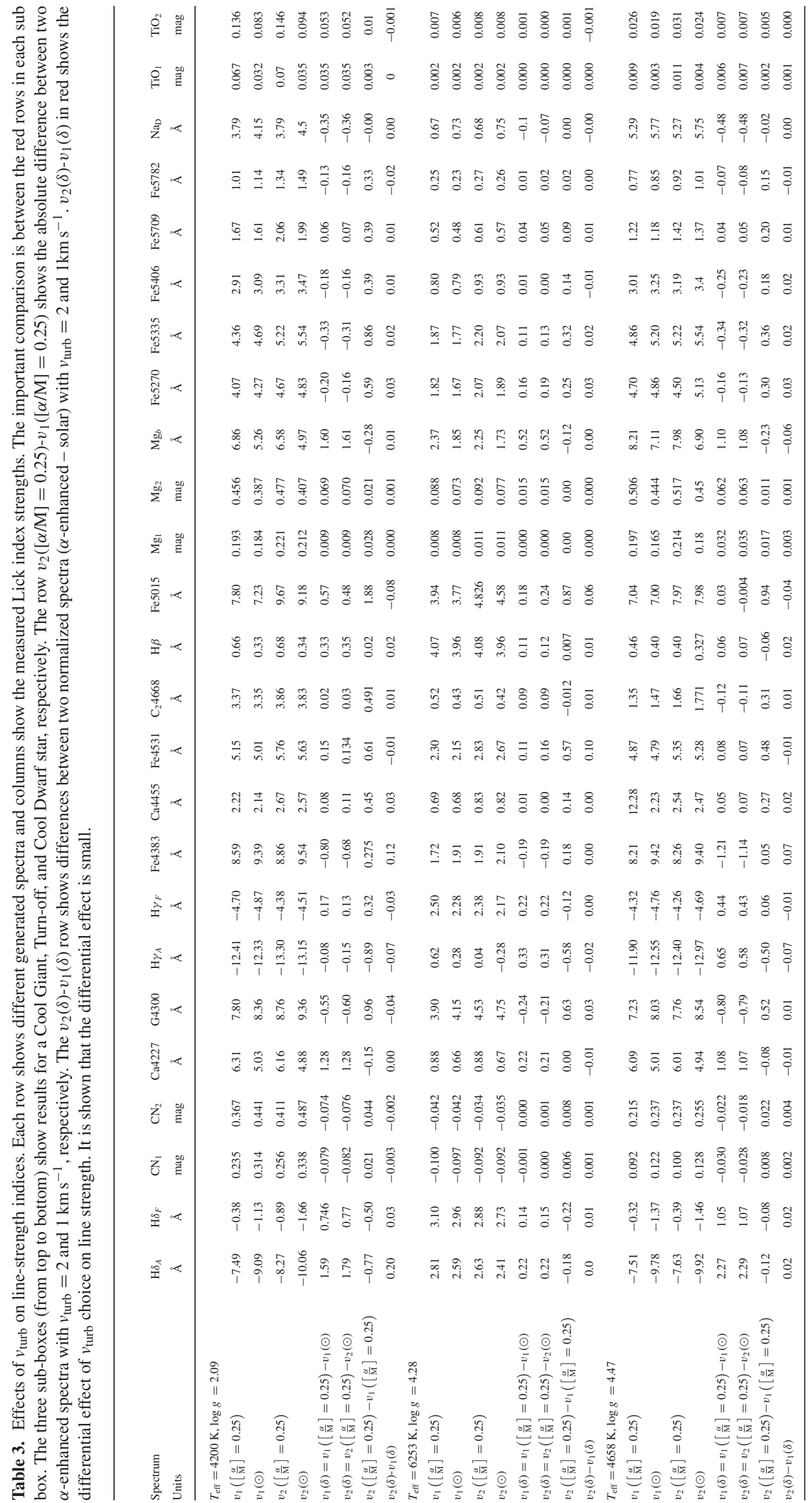



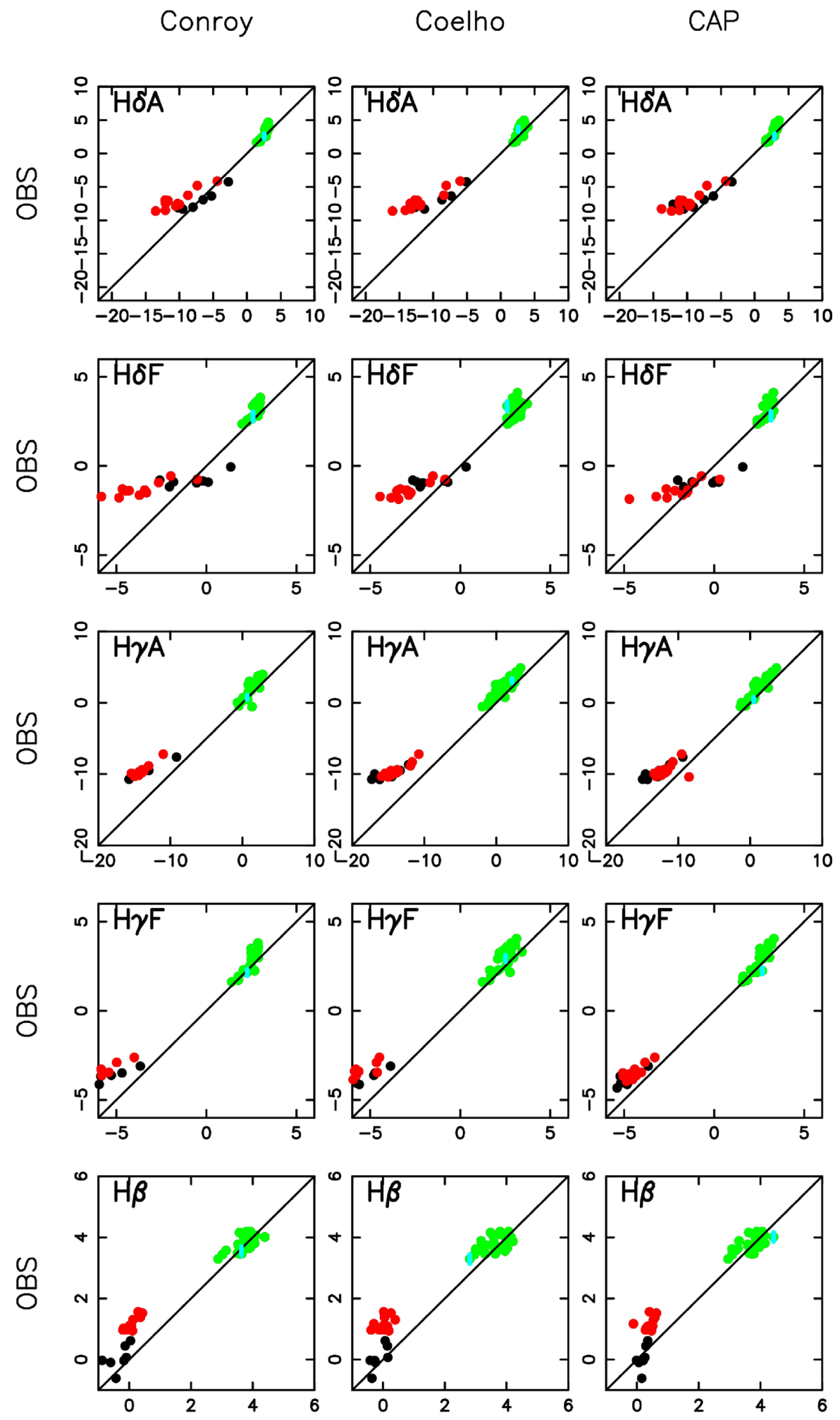

MODEL

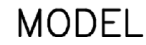

MODEL

Figure 1. MILES Lick Indices versus Model Lick Indices, for Conroy, Coelho, and Allende Prieto (CAP) theoretical spectra that match the MILES atmospheric parameters given in Cenarro et al. (2007), for hydrogen-sensitive features. The three star types are shown in each case, with green, black, and red circles representing Turn-Off, Cool Dwarf, and Cool Giant stars, respectively. 

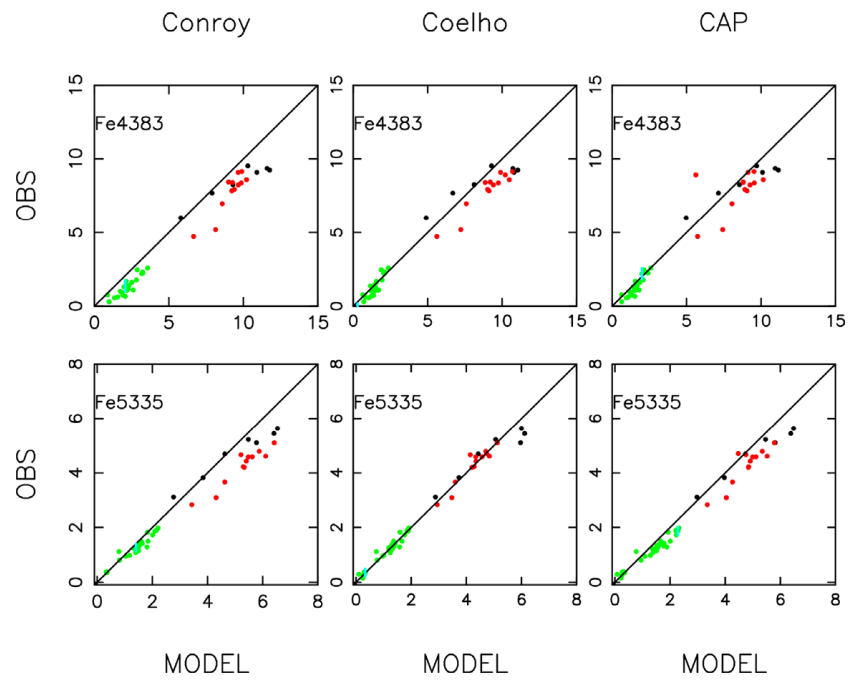

Figure 2. MILES Lick Indices versus Model Lick Indices, for CAP theoretical spectra, respectively, for iron-sensitive features. Same parameters and labelling procedure as Fig. 1.
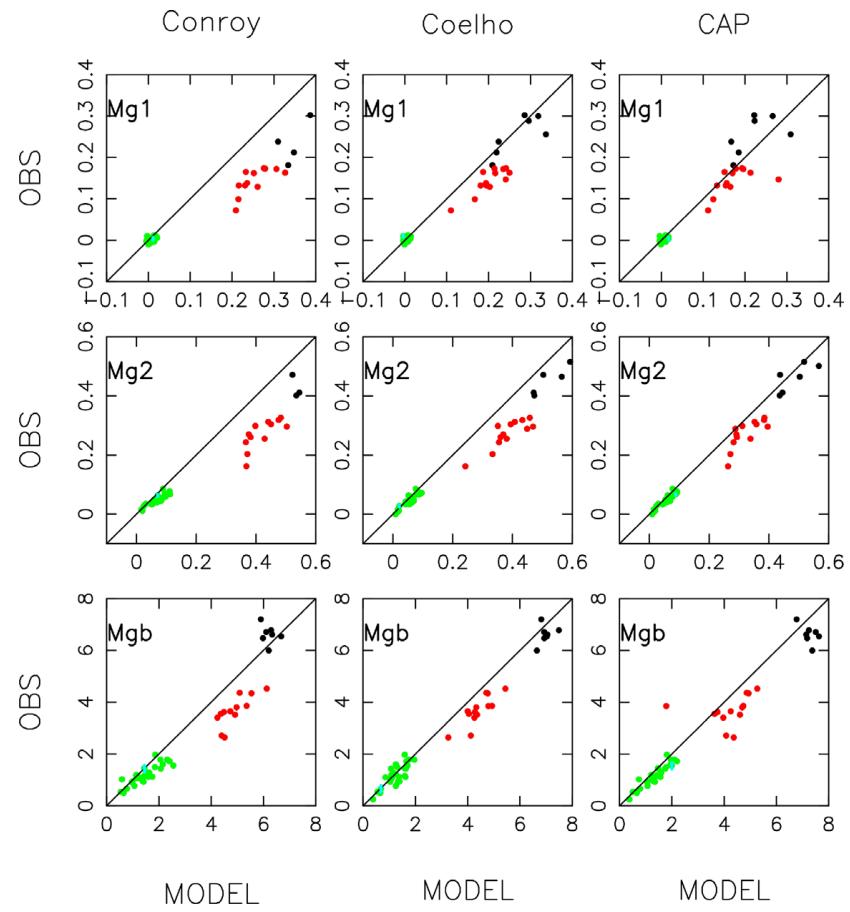

Figure 3. MILES Lick Indices versus Model Lick Indices, for CAP theoretical spectra, respectively, for magnesium-sensitive features. Same parameters and labelling procedure as Fig. 1.

approach attempts to isolate the effects of abundance and abundance pattern only. The base model parameters are shown in Section 2. The MILES Cool Dwarf, Turn-off, and Cool Giant base stars are HD 032147, HD 016673, and HD 154733, respectively. The parameters of these stars are shown in Sansom et al. (2013, table 3).

To derive the theoretical response functions, the model spectra were matched to MILES resolution and sampling. They were resampled from a log scale to a linear scale, taking the largest wavelength interval of the raw theoretical spectrum as the linear sampling. The theoretical spectra were then degraded and resampled to match the MILES observations, as described in Section 4. The

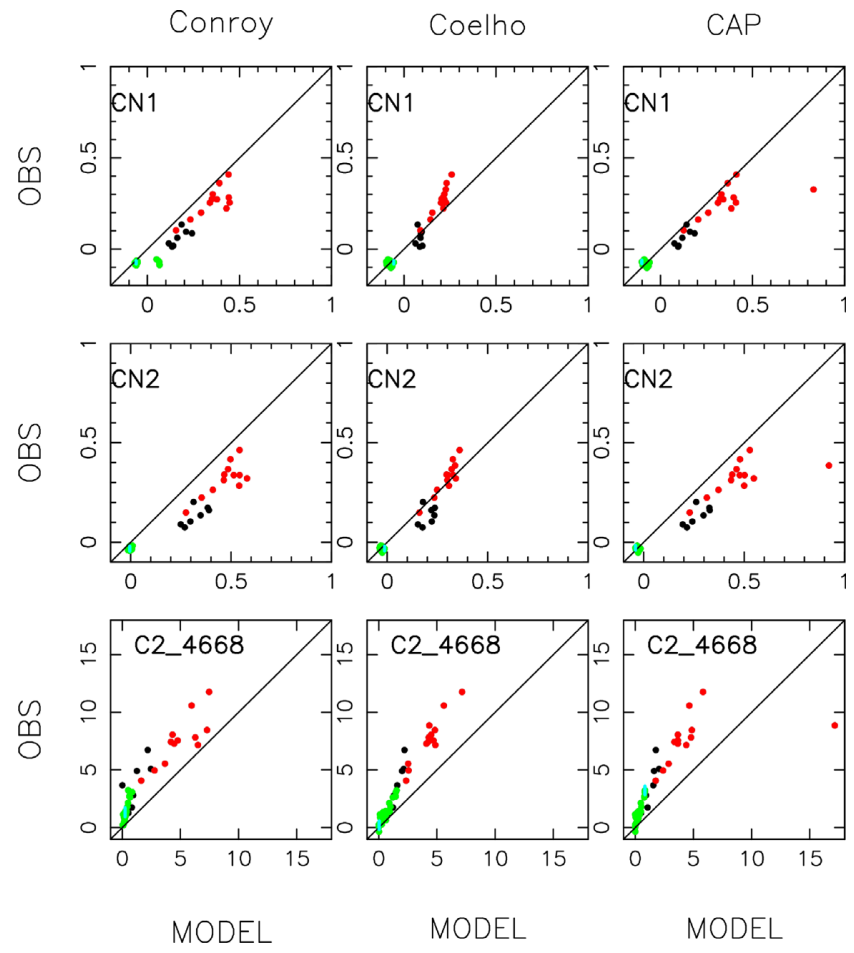

Figure 4. MILES Lick Indices versus Model Lick Indices, for CAP theoretical spectra, respectively, for carbon-sensitive features. Same parameters and labelling procedure as Fig. 1. The outlier point in the CAP model plots is HD131430, with parameters $T_{\text {eff }}=4190 \mathrm{~K}, \log g=1.95,[\mathrm{Fe} / \mathrm{H}]=0.1$, and $[\mathrm{Mg} / \mathrm{Fe}]=-0.398$.
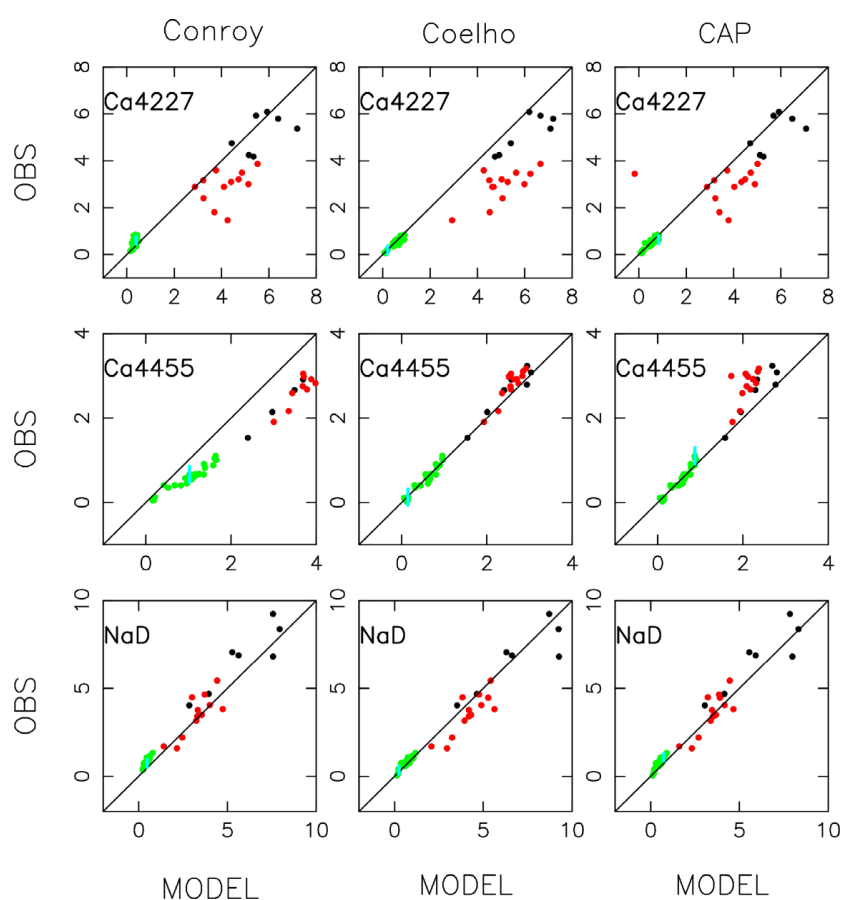

Figure 5. MILES Lick Indices versus Model Lick Indices, for CAP theoretical spectra, respectively, for calcium- and sodium-sensitive features. Same parameters and labelling procedure as Fig. 1. 
25 Lick line-strength indices were then measured using LECTOR. Individual response functions for the three star types were derived by finding the differences of indices, relative to solar abundance pattern, for the element enhanced spectra of each star type. For example, to calculate the magnesium response function for a Cool Dwarf spectrum, we take the difference in indices between the $\mathrm{Mg}+0.3$ enhanced spectrum and solar abundance pattern spectrum. This is then repeated for all the element enhanced spectra provided, in order to derive the response functions for individual element changes and for overall metallicity changes. We then apply the response functions to account for changes in abundances as described below.

In the application of response functions, we make the typical assumption that absorption-line strengths are linearly proportional to the number of absorbers. We follow the methodology presented in Sansom et al. (2013), which is based on the works of Thomas, Maraston \& Bender (2003) and Korn et al. (2005). We account for indices that go negative by conserving flux, as described in equation 3 of Korn et al. (2005). We tested the reliability of interpolating response functions by computing a Cool Giant star for CAP models at intermediate $[\alpha / \mathrm{Fe}]$ values (e.g. $[\alpha / \mathrm{Fe}]=0.2)$ and comparing the model Lick indices to those produced by applying response functions from each of the $\alpha$ elements individually. Apart from three outlier indices $\left(\mathrm{Ca} 4227, \mathrm{C}_{2} 4668\right.$, and $\left.\mathrm{TiO}_{1}\right)$, we find good agreement between the two methods, with an RMS scatter of 0.07 , for indices that are measured in $\AA$. This is within typical index measurement errors. Investigation into the outlier indices found that the problem is due to both side and feature bands of the Lick index being affected by a total $[\alpha / \mathrm{Fe}]$ enhancement, which does not match the effects caused by changing the $\alpha$ elements separately. However, because the majority of the MILES stars used in this study have an $[\mathrm{Mg} / \mathrm{Fe}]$ value much less than 0.3 , the application of response functions in this range is reliable.

Due to the lack of MILES stars with combinations of $T_{\text {eff }}, \log g$, and $[\mathrm{Fe} / \mathrm{H}]$ to match the theoretical stars provided at solar $[\mathrm{Fe} / \mathrm{H}]$, the derived response functions are applied twice to the base model star indices. First, a correction is made to match the model to the equivalent MILES star in $[\mathrm{Fe} / \mathrm{H}]$ using the $[\mathrm{M} / \mathrm{H}]$ column of the response function (see Table 4). Second, a correction is made to reach the correct $[\alpha / \mathrm{Fe}]$ using the $\alpha$ element columns. The $\alpha$ elements used in each case are specified in Section 2.

The $[\alpha / \mathrm{Fe}]$-enhanced, or deficient, star indices are normalized by the corresponding solar abundance pattern base model $\left(\mathrm{TI}_{\odot}\right)$ or base MILES star $\left(\mathrm{OI}_{\odot}\right)$ indices through divisions given in equations (2) and (3). Non-solar $[\alpha / \mathrm{Fe}]$ MILES or Model indices are referred to as $\mathrm{OI}_{\alpha}$ and $\mathrm{TI}_{\alpha}$, respectively. We refer to an MILES or Model normalized index as OBS/BASE or MODEL/BASE_MODEL, respectively:

$\mathrm{OBS} / \mathrm{BASE}=\frac{\mathrm{OI}_{\alpha}}{\mathrm{OI}_{\odot}}$

MODEL/BASE_MODEL $=\frac{\mathrm{TI}_{\alpha}}{\mathrm{TI}_{\odot}}$

For molecular bands and weak-line features that tend to zero or are negative, the normalization process is performed via a difference rather than a ratio.

$\mathrm{OBS}-\mathrm{BASE}=\mathrm{OI}_{\alpha}-\mathrm{OI}_{\odot}$,

MODEL - BASE_MODEL $=\mathrm{TI}_{\alpha}-\mathrm{TI}_{\odot}$.
Complete agreement between the observations and predictions from theoretical response functions would lead to a ratio of MILES Normalized Index $=$ Model Normalized Index.

Figs 6-10 show the comparison of normalized Lick indices derived from MILES stars to those derived from predictions of the theoretical response functions, for selected Lick indices. These figures highlight the main effects that we found. Observational errors on indices were estimated per star type, considering twice the random errors. Selecting a larger sample of MILES cool stars to calculate the random errors, we find that the errors increase by a factor of $\sim 40$ per cent compared to the error calculated from just the 13 Cool Giant stars. There is at least a factor of $\sqrt{2}$ because both the enhanced and the base star are affected in the normalized indices. That is why, we have used a conservative value of twice the random errors. Systematic errors due to atmospheric parameter uncertainties were estimated for each star type using the online MILES interpolator. ${ }^{5}$ Note that errors in the atmospheric parameters of the base star would lead to systematic offsets in differences and systematic deviations in the slope in ratios. In the following plots, stars with $[\mathrm{Fe} / \mathrm{H}]<-0.4$ (represented by open symbols) sometimes fall outside of the ranges of the plots, particularly in the blue end of the spectrum, with two Cool Dwarfs, one Cool Giant, and one Turn-off star affected. Another outlier is a Cool Giant with $[\mathrm{Mg} / \mathrm{Fe}]=-0.398$ (HD131430). This is likely to be uncertain because the calibration used in Milone et al. (2011) (their fig. 4) did not extend to such low values in $[\mathrm{Mg} / \mathrm{Fe}]$. This star is an outlier in $\mathrm{CN}_{1}$ and $\mathrm{CN}_{2}$ of the CAP and Coelho models.

Fig. 6 shows the response function comparison of models versus empirical stars, respectively, for Hydrogen Lick indices. We find a disagreement in Turn-off stars for all models, with empirical stars showing a larger range of variation than predicted in the models, particularly for $\mathrm{H} \gamma_{A}$. There is the opposite behaviour in models for the cool stars in Fig. 6. All three models appear to overpredict the variation in the $\mathrm{H} \delta_{\mathrm{A}}$ and $\mathrm{H} \delta_{\mathrm{F}}$ indices in both Cool Dwarf and Cool Giant stars. This is the same trend in cool stars as found for Korn et al. (2005) models, in Sansom et al. (2013; see their fig. 1b). The models perform better for the $\mathrm{H} \gamma_{\mathrm{A}}$ and $\mathrm{H} \gamma_{\mathrm{F}}$ indices, lying closer to the 1:1 line for Conroy, and furthest for Coelho. Conroy's Cool Dwarf models predict almost no variation in $\mathrm{H} \gamma_{\mathrm{F}}$ for changes in abundance pattern, highlighted by the almost vertical pattern seen in the plot. Variation in the $\mathrm{H} \beta$ index shows no clear trends. We investigate a different definition of the $\mathrm{H} \beta$ index, $\mathrm{H} \beta_{0}$, in Appendix B. For $\mathrm{H} \beta_{0}$, we find a stronger correlation with abundance pattern and metallicity in $\mathrm{H} \beta_{0}$ for all models, which is in agreement with the theoretical SSPs of Cervantes \& Vazdekis (2009). In summary, for all indices, there is a general lack of agreement for cool stars in all three models, with some improvements seen in Conroy and CAP models.

Fig. 7 shows the comparison between model predictions and MILES stars for two iron-sensitive features. Other iron-sensitive features show similar agreement. This highlights that all iron model response function predictions for all star types agree well with the MILES stars.

Fig. 8 shows predictions of the models for Mg-sensitive indices. The scatter is quite large. All models show generally the same behaviour - the Cool Giant and Turn-off models all systematically overpredict the strength in Fig. 8, lying below the 1:1 line. The Cool Dwarf models show a good agreement with the 1:1 line in these $\mathrm{Mg}$-sensitive features. 
Table 4. Extract from Response Functions. CAP Cool Giant stars. Column 1 is the Lick index name, Column 2 is the units of the index, Column 3 is the model base star index strength, and Columns 4-13 are the variation of the index strength (in units of mag or $\AA$ ) when the element at the top of the column is increased by 0.3 dex above solar. The last column shows the variation of the index strength when there is an overall metallicity increase of 0.3 dex. Full versions of this and all the derived response functions can be found in the online supplementary material and at http://uclandata.uclan.ac.uk/id/eprint/175.

\begin{tabular}{|c|c|c|c|c|c|c|c|c|c|c|c|c|c|}
\hline Index & Units & $\mathrm{I}_{0}$ & $\mathrm{C}$ & $\mathrm{N}$ & $\mathrm{O}$ & $\mathrm{Mg}$ & $\mathrm{Fe}$ & $\mathrm{Ca}$ & $\mathrm{Na}$ & $\mathrm{Si}$ & $\mathrm{Cr}$ & $\mathrm{Ti}$ & {$[\mathrm{M} / \mathrm{H}]$} \\
\hline $\mathrm{H} \delta_{\mathrm{A}}$ & $\AA$ & -10.125 & -2.337 & 0.007 & 0.865 & 0.966 & -2.010 & -0.085 & -0.003 & 1.768 & 0.151 & -0.321 & -1.433 \\
\hline $\mathrm{H} \delta_{\mathrm{F}}$ & $\AA$ & -1.922 & -1.071 & -0.156 & 0.225 & 0.074 & -1.025 & 0.031 & -0.017 & 1.267 & -0.014 & -0.254 & -0.794 \\
\hline $\mathrm{CN}_{1}$ & mag & 0.341 & 0.510 & 0.089 & -0.108 & -0.053 & -0.057 & -0.010 & -0.005 & 0.022 & -0.016 & 0.002 & 0.040 \\
\hline $\mathrm{CN}_{2}$ & mag & 0.461 & 0.517 & 0.089 & -0.110 & -0.059 & -0.064 & -0.012 & -0.004 & 0.049 & -0.014 & 0.002 & 0.046 \\
\hline $\mathrm{Ca} 4227$ & $\AA$ & 4.111 & -0.866 & -0.172 & 0.386 & 0.439 & 0.266 & 1.897 & 0.091 & -0.010 & -0.040 & -0.023 & 1.430 \\
\hline$\ldots$ & $\ldots$ & $\ldots$ & $\ldots$. & $\ldots$ & $\ldots$ & $\ldots$ & $\ldots$ & $\ldots$ & $\ldots$ & $\ldots$ & $\ldots$ & $\ldots$ & \\
\hline
\end{tabular}

Fig. 9 shows predictions of the models for carbon-sensitive indices. There are systematic deviations from the 1:1 line in the $\mathrm{CN}$ features of Conroy's models, with a smaller range of differential behaviour in cool stars with a larger range of differential behaviour of $\mathrm{CN}_{2}$ in metal-poor Turn-off stars, compared to MILES stars. The differential predictions for the $\mathrm{CN}$ features of both Coelho and CAP models are similar, with a good agreement with the 1:1 lines.

For the $\mathrm{C}_{2} 4668$ feature shown in Fig. 9, the differential predictions of the Turn-off stars show good agreement between observations and models for the Coelho and CAP cases, but less so for the Conoy case. The cool star models of Coelho and CAP are in good agreement with the 1:1 lines. In the Conroy models, there are four outlier Cool Dwarf stars that fall outside of the plot. This is caused by a problem with the base model, which has a different index to the ones found in real stars. Conroy's base model index was -0.105 , compared to 1.453 and 1.406 of Coelho and CAP models, respectively.

Fig. 10 shows the response function predictions for calcium- and sodium-sensitive indices. This shows that all models predict the differential behaviour of $\mathrm{Ca} 4455, \mathrm{Ca} 4227$, and $\mathrm{Na}_{\mathrm{D}}$ features quite well, with the models lying close to, the 1:1 line.

Models are assessed via reduced chi-square $\left(\chi_{v}^{2}\right)$ analysis. Table 5 shows $\chi_{v}^{2}$ values, about the 1:1 agreement line, for the normalized Cool Giant models versus normalized MILES observations. The values calculated took into account the errors associated with the observations and systematic offsets caused by atmospheric parameter uncertainties added in quadrature. The calculations were performed using all stars with $[\mathrm{Fe} / \mathrm{H}]>-0.4$, apart from a Cool Giant star outlier (HD131430). With the $[\mathrm{Fe} / \mathrm{H}]$ cut and removal of HD131430, this left 11 Cool Giant stars for the calculation. We also assess the performance of models via linear regression between normalized index observations and model predictions. Table 6 shows the results for Cool Giant models, indicating the derived gradient and intercept for the best-fitting linear trends. The results from this regression highlight the differences between the model trends and 1:1 agreement. With such a fit, if the model is agreeing fully with the observations we expect to find a gradient of 1 and intercept of 0 . The combination of $\chi_{v}^{2}$, gradient and intercept gives information about any scatter or offsets of the models from the $1: 1$ agreement line.

We find that there is a lack of agreement between cool star models and observations for Balmer features, with the $\chi_{v}^{2}$ showing that for $\mathrm{H} \delta_{\mathrm{A}}, \mathrm{H} \gamma_{\mathrm{A}}$, and $\mathrm{H} \gamma_{\mathrm{F}}$ Coelho models are performing the worst, improvements are seen in CAP and Conroy is performing the best. Coelho models show the shallowest gradients for these $\mathrm{H} \delta_{\mathrm{A}}$ and $\mathrm{H} \gamma_{\mathrm{A}}$ features. The $\mathrm{C}_{2} 4668$ results show that all the Cool Giant models have very similar predictions for this index. From the $\mathrm{Mg}_{b}$ results, there is a much larger scatter found than expected. This behaviour is also true for Ca4227, with large $\chi_{v}^{2}$ values found. Reflecting the results shown in Fig. 7, the $\chi_{v}^{2}$, gradient and intercept values highlight that iron features are fit well by the models. Considering the poorer fits, where $\chi_{v}^{2}>1\left(\mathrm{H} \delta_{\mathrm{A}}, \mathrm{H} \delta_{\mathrm{F}}, \mathrm{Ca} 4227, \mathrm{H} \gamma_{\mathrm{F}}\right.$, $\left.\mathrm{Ca} 4455, \mathrm{C}_{2} 4668, \mathrm{Mg}_{\mathrm{b}}, \mathrm{Fe} 5335\right)$, we summarize that regarding the Cool Giant models, Conroy models perform best in three out of those eight indices, and perform worst in two of them; Coelho models perform best in two, and worst in three indices; CAP models perform best in three and worst in three indices. For the other five indices $\left(\mathrm{CN}_{1}, \mathrm{CN}_{2}, \mathrm{H} \gamma_{\mathrm{A}}, \mathrm{Fe} 4383\right.$, and $\left.\mathrm{H} \beta\right)$ all the models fit the data $\left(\chi_{v}^{2}<1\right)$. Cool Dwarf models show similar behaviour to the Cool Giant models, whereas Turn-off models all have $\chi_{v}^{2}<1$ except for $\mathrm{C}_{2} 4668$ and $\mathrm{Mg}_{\mathrm{b}}$. Regarding the gradients shown in Table 6 for red giant stars, Conroy models have seven indices, Coelho models have seven indices, and CAP models have nine indices, with a gradient in the range $0.5-1.5$ of the $1: 1$ line.

\section{DISCUSSION}

There are two main caveats to our analysis. First, we have used MILES atmospheric parameters presented in Cenarro et al. (2007) throughout this paper. Therefore, the effects of alternative stellar parameters have not been studied (e.g Sharma, Prugniel \& Singh 2016). Secondly, we have not attempted to address other departures from solar-scaled patterns (such as $\mathrm{C}, \mathrm{N}$, and $\mathrm{O}$ ), which might affect the empirical stars but are not accounted for in the models. These departures may affect the absolute predictions more than differential predictions, compared later in this section. The effects of $\mathrm{C}, \mathrm{N}$, and $\mathrm{O}$ abundances are beyond this study due to the lack of abundance information currently available for MILES stars.

We will now discuss the main deviations found from the index analysis performed in Sections 4 and 5.

\subsection{Indices}

We discuss the largest disagreements found between models in more detail, focusing on hydrogen-sensitive features. We also show the differences between using the models differentially and absolutely.

\subsubsection{Hydrogen indices}

The cause of deviations between observed and model predictions for $\mathrm{H}_{\gamma}$ and $\mathrm{H}_{\delta}$ features appears to be related to temperature, with increasing disagreement at lower star temperature. Lowtemperature stars are known to be difficult to model accurately, due to the complexity of absorption features in their atmospheres. The models tested here are generated using versions of ATLAS therefore, 
Conroy

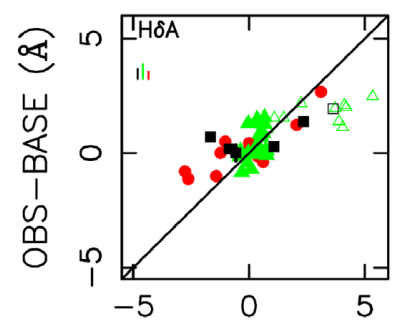

MODEL-BASE_MODEL

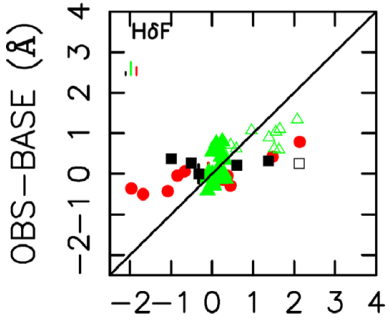

MODEL-BASE_MODEL

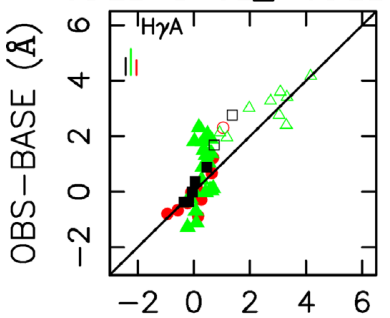

MODEL-BASE_MODEL

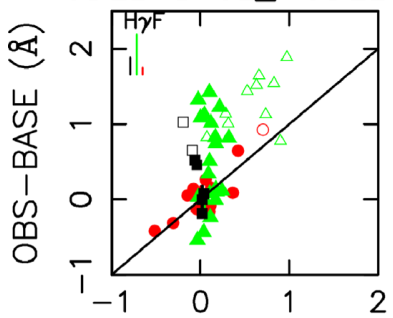

Coelho

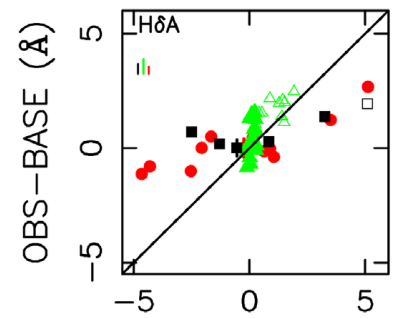

(§) MODEL-BASE_MODEL

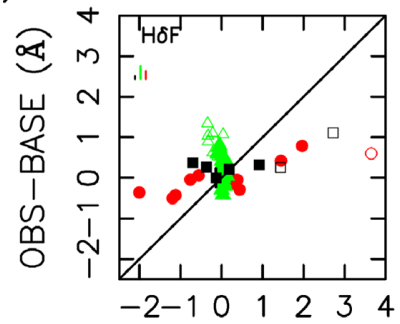

(\&)

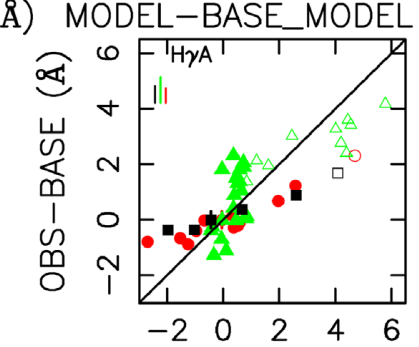

(§) MODEL-BASE_MODEL

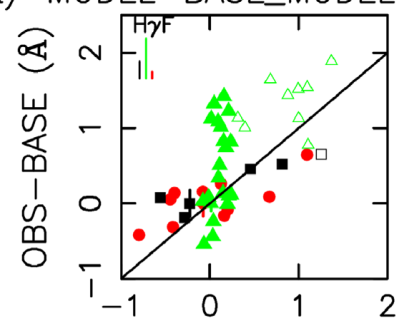

CAP

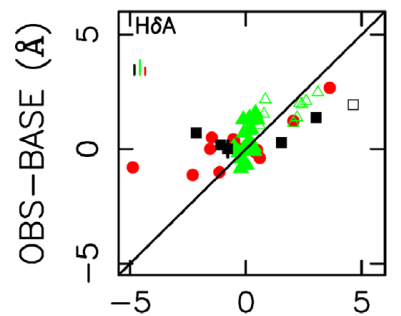

( $\AA)$ MODEL-BASE_MODEL $(\AA)$

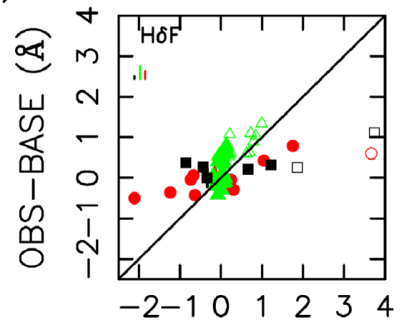

( $\AA)$ MODEL-BASE_MODEL ( $\AA)$

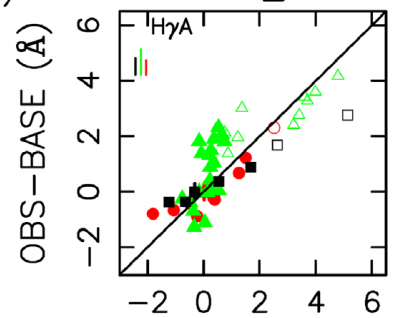

( $\AA)$ MODEL-BASE_MODEL ( $\AA)$

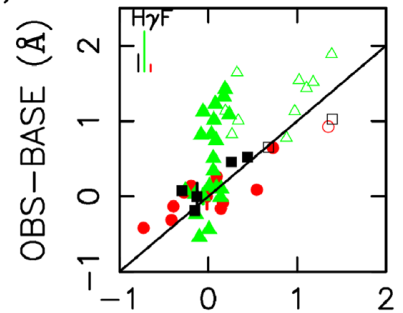

MODEL-BASE_MODEL

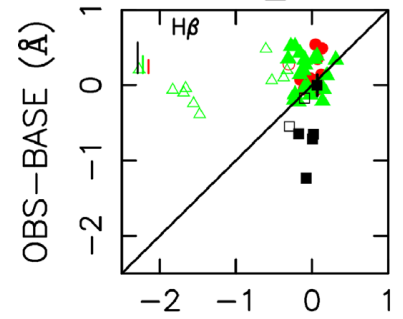

(§) MODEL-BASE_MODEL

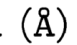
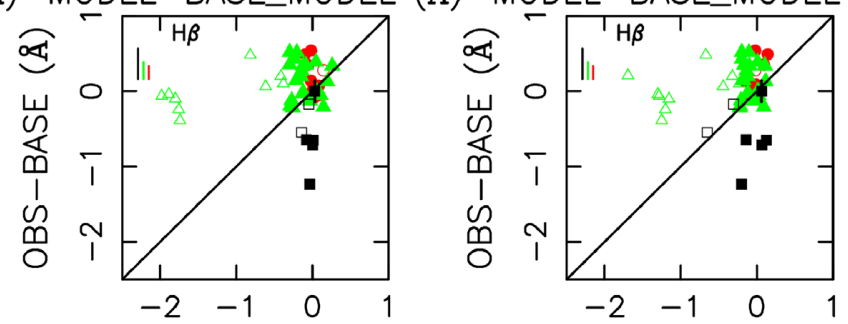

MODEL-BASE_MODEL

(§) MODEL-BASE_MODEL

$(\AA)$

MODEL-BASE_MODEL ( ()

Figure 6. MILES Normalized Lick indices versus CAP Model Normalized Lick indices derived from response functions, for Hydrogen indices. The three star types are shown in each case, with green triangles, black squares, and red circles representing Turn-Off, Cool Dwarf, and Cool Giant stars, respectively. Open symbols represent stars with $[\mathrm{Fe} / \mathrm{H}]<-0.4$. The observational error bar is shown on the corresponding base star point in each plot and $+1 \sigma$ error bars due to star parameter uncertainities are shown top left in each plot. 

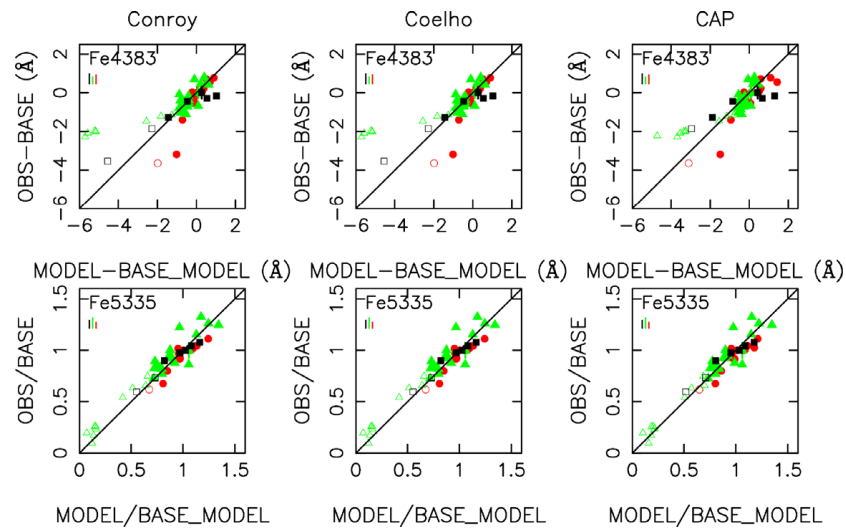

Figure 7. MILES Normalized Lick indices versus CAP Model Normalized Lick indices, for iron-sensitive features. Symbols and colours as in Fig. 6.
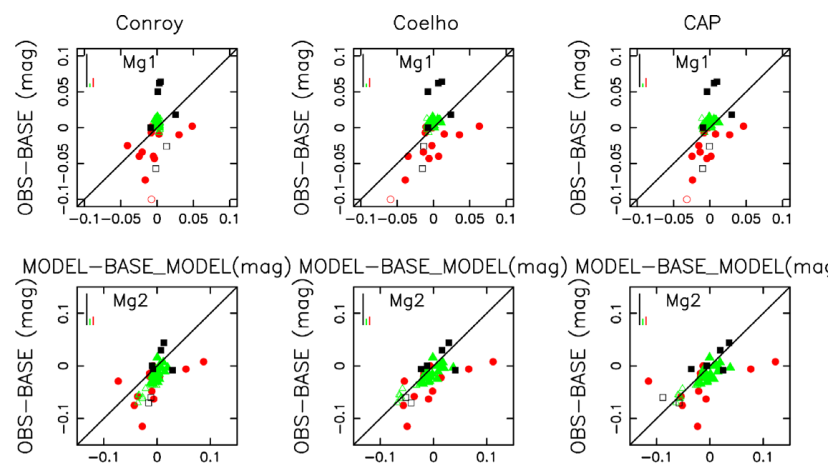

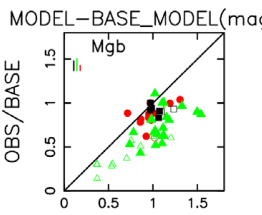

MODEL/BASE_MODEL

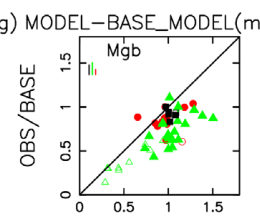

MODEL/BASE_MODEL

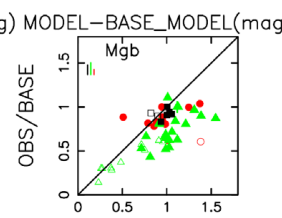

MODEL/BASE_MODEL
Figure 8. MILES Normalized Lick indices versus CAP Model Normalized Lick indices, for Magnesium $\left(\mathrm{Mg}_{1}, \mathrm{Mg}_{2}\right.$, and $\left.\mathrm{Mg}_{b}\right)$-sensitive features. Symbols and colours as in Fig. 6.

spherical geometry and non-LTE effects have been ignored. This will impact the lowest temperatures and may explain the lack of agreement between models and observations for the cool star models. The models overpredict variations in $\mathrm{H}_{\gamma}$ and $\mathrm{H}_{\delta}$ features compared to observations. Where these features are used for age indicators in stellar populations, age estimates will be affected. In the absolute comparisons, where the models underpredict the strength of hydrogen lines (Fig. 1, top four rows), the ages of stellar populations would be overestimated. In the differential comparisons, where the models both under- and overpredict the hydrogen lines (Fig. 6, top four rows), the ages of stellar populations could be over- or underpredicted.

\subsubsection{Differential versus absolute indices}

In this section we present quantitative results that highlight the differences in reliability between using theoretical models in a differential way and using their absolute predictions. In Fig. 11 we show an alternative way to present this difference for the 11
CAP Cool Giant models versus the MILES giant stars in our 51 star sub-sample.

In Fig. 11 we show the difference in theoretical and observational Lick indices versus wavelength for the 19 indices that are in units of $\AA$. For the red points (open circles), Theory = Theoretical Enhanced Index - Theoretical Base Index and Obs = Observational Enhanced Index - Observational Base index. For the blue points (stars), Theory $=$ Theoretical Enhanced Index and Obs = Observational Enhanced Index. The difference in location between the red points and blue points highlights the effect of normalizing the enhanced indices by corresponding solar abundance pattern indices. In general, the differential predictions (red points) are far less scattered about the Theory = Obs line than the blue points, particularly in the blue part of the spectrum (below $\mathrm{Mg}_{\mathrm{b}}$ ). The differential approach generally appears to produce more reliable predictions than the absolute predictions, which is highlighted in Table 7, where we show the RMS scatter for the blue and red points in the case of Theory-Obs. For the differential approach, almost all of the indices are scattered less or the same as the absolute predictions about the Theory $=$ Obs line. The results in Table 7 show that the differential application of theoretical stellar spectra produces generally a better prediction of abundance pattern effects than the absolute. This is highlighted with the large ( $\sim$ factors of 2 or more) improvements in RMS scatter of the $\mathrm{H} \delta_{\mathrm{A}}, \mathrm{G} 4300, \mathrm{H} \gamma, \mathrm{Fe} 4383, \mathrm{Ca} 4455, \mathrm{C}_{2} 4668$, and $\mathrm{H} \beta$ indices. For the two carbon-sensitive features, G4300 and $\mathrm{C}_{2} 4668$, the lack of carbon information in MILES stars may explain the poor absolute predictions of models in Table 7.

In Table 7 we also show the RMS scatter when assuming a zero $\alpha$ response (e.g. we only apply the response functions to match the MILES $[\mathrm{Fe} / \mathrm{H}]$ values, not the $[\alpha / \mathrm{Fe}]$ as well). Interestingly, it can be seen that almost all the indices are as good as or better than the differential approach that matched both $[\mathrm{Fe} / \mathrm{H}]$ and $[\alpha / \mathrm{Fe}]$ values. This highlights the large metallicity dependence of these indices, with the $\alpha$ response being a secondary effect. Therefore, we conclude that this assessment, using response functions, does not test abundance patterns as well as expected, because of the small range of $[\mathrm{Mg} / \mathrm{Fe}]$ in the Cool Giant MILES stars used in this study.

\subsection{Synthetic spectra}

To highlight comparisons between models, we plot examples of spectral ratios in regions of Lick indices. Using the stars generated for the direct comparison in Section 4, we investigate the differences between normalized (non-solar abundance pattern star/Base star) cool giant empirical and model spectra. The stars we chose were HD113092 $([\mathrm{Fe} / \mathrm{H}]=-0.370,[\mathrm{Mg} / \mathrm{Fe}]=0.182)$ and HD154733 $([\mathrm{Fe} / \mathrm{H}]=-0.080,[\mathrm{Mg} / \mathrm{Fe}]=0.009) . \mathrm{HD} 154733$ was the Cool Giant base star used in the analysis in Section 5. The ratio of these two stars shows the differential effect of both metallicity and $\alpha$ abundance changes. We focus on indices showing the largest disagreements between model and observations.

Fig. 12 highlights spectral differences in the $\mathrm{C}_{2} 4668$ region between the CAP and Coelho models. Normalized CAP models are offset from the normalized observations, which is not seen in the differential index analysis. On the other hand, normalized Coelho models lie closer to the normalized observations. These offsets of spectra appear to have little effect on the differential Lick indices. This is seen in Fig. 9, where both Cool Giant models show good agreement with the observations in the $\mathrm{C}_{2} 4668$ index.

Fig. 13 highlights some of the problems with the higher order Balmer features seen in Fig. 6. Both Coelho and CAP models are overpredicting features in $\mathrm{H} \delta_{\mathrm{A}}$, in pseudocontinua and feature 
Conroy

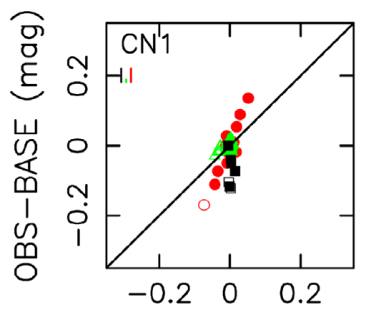

Coelho

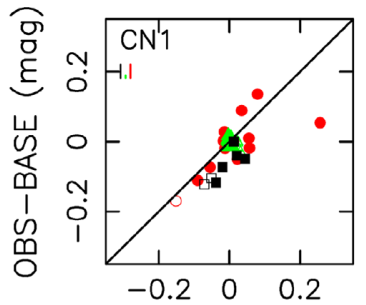

CAP

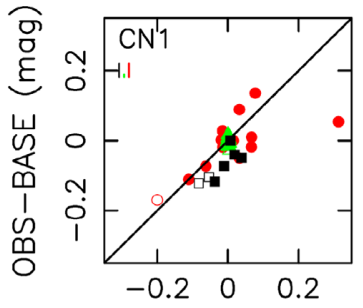

MODEL-BASE_MODEL(mag) MODEL-BASE_MODEL(mag) MODEL-BASE_MODEL(mag)
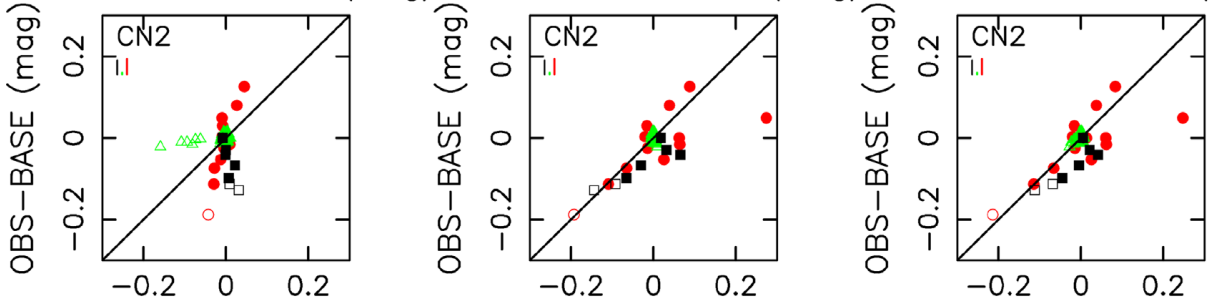

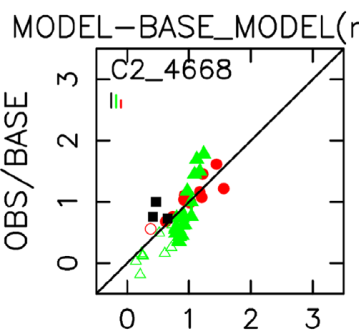

MODEL/BASE_MODEL

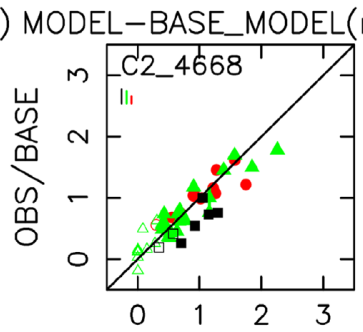

MODEL/BASE_MODEL

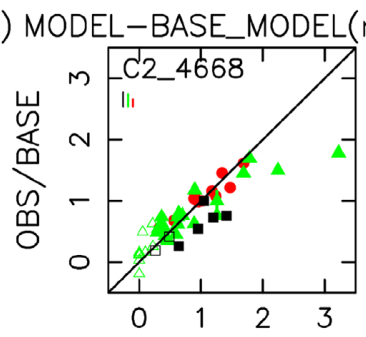

MODEL/BASE_MODEL

Figure 9. MILES Normalized Lick indices versus CAP Model Normalized Lick indices, for carbon- and nitrogen-sensitive features. Four cool dwarf stars fall outside the range of the Conroy $\mathrm{C}_{2} 4688$ plot. Symbols and colours as in Fig. 6.
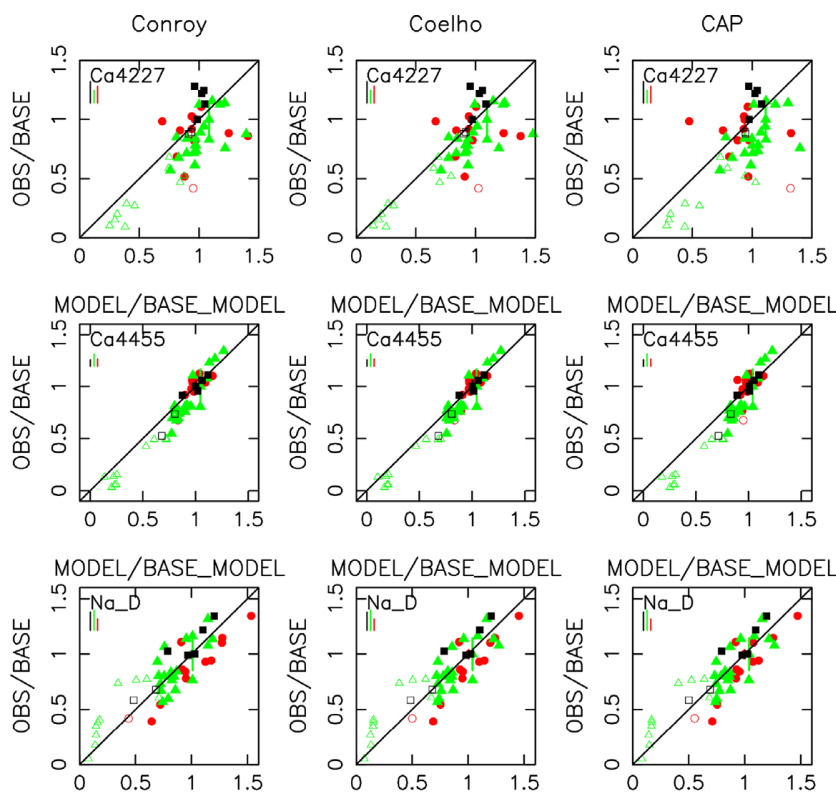

MODEL/BASE_MODEL

MODEL/BASE_MODEL
Figure 10. MILES Normalized Lick indices versus CAP Model Normalized Lick indices, for calcium- and sodium-sensitive features. Symbols and colours as in Fig. 6.
Table 5. Reduced $\chi_{v}^{2}$ values for comparisons of normalized observations (from MILES) versus normalized models (using the response functions for $[\mathrm{M} / \mathrm{H}]$ and $[\alpha / \mathrm{Fe}]$ changes in stars). The results are shown for Cool Giant stars and for star response functions from Conroy, Coelho, and CAP. These values were calculated for stars with $[\mathrm{Fe} / \mathrm{H}]>-0.4$ and with $\mathrm{HD} 131430$ removed (see Section 5 for details).

\begin{tabular}{lccc}
\hline Index & $\begin{array}{c}\text { Conroy } \\
\chi_{v}^{2}\end{array}$ & $\begin{array}{c}\text { Coelho } \\
\chi_{v}^{2}\end{array}$ & $\begin{array}{c}\text { CAP } \\
\chi_{v}^{2}\end{array}$ \\
\hline $\mathrm{H} \delta_{\mathrm{A}}$ & 1.44 & 6.21 & 1.92 \\
$\mathrm{H} \delta_{\mathrm{F}}$ & 3.24 & 3.01 & 1.51 \\
$\mathrm{CN}_{1}$ & 0.32 & 0.33 & 0.40 \\
$\mathrm{CN}_{2}$ & 0.36 & 0.35 & 0.35 \\
$\mathrm{Ca} 4227$ & 7.12 & 6.94 & 10.13 \\
$\mathrm{H} \gamma_{\mathrm{A}}$ & 0.07 & 0.80 & 0.21 \\
$\mathrm{H} \gamma_{\mathrm{F}}$ & 0.56 & 2.26 & 1.09 \\
$\mathrm{Fe} 4383$ & 0.55 & 0.36 & 0.39 \\
$\mathrm{Ca} 4455$ & 0.75 & 0.69 & 1.06 \\
$\mathrm{C}_{2} 4668$ & 5.06 & 5.12 & 4.56 \\
$\mathrm{H} \beta$ & 0.35 & 0.56 & 0.36 \\
$\mathrm{Mg}$ & 19.13 & 19.90 & 26.26 \\
$\mathrm{Fe} 5335$ & 5.05 & 4.87 & 4.61 \\
\hline
\end{tabular}

bands. This overprediction may contribute to trends seen in Fig. 6, with cool star models showing more variation in the index than in observed stars. These type of plots allowed us to identify that the file regarding the transition D-A of the molecule $\mathrm{C}_{2}$ in Coelho (2014) 
Table 6. Gradient and intercept values calculated from a linear regression for comparisons of normalized observations (from MILES) versus normalized models (using the response functions for $[\mathrm{M} / \mathrm{H}]$ and $[\alpha / \mathrm{Fe}]$ changes in stars). The results are shown for Cool Giant stars and for star response functions from Conroy, Coelho, and CAP. These values were calculated for stars with $[\mathrm{Fe} / \mathrm{H}]>-0.4$ and with $\mathrm{HD} 131430$ removed.

\begin{tabular}{lcccrrr}
\hline & \multicolumn{2}{c}{ Conroy } & \multicolumn{2}{c}{ Coelho } & & CAP \\
Index & Gradient & Intercept & Gradient & Intercept & Gradient & Intercept \\
\hline $\mathrm{H} \delta_{\mathrm{A}}$ & $0.55 \pm 0.12$ & $0.19 \pm 0.18 \AA$ & $0.32 \pm 0.07$ & $0.19 \pm 0.18 \AA$ & $0.50 \pm 0.12$ & $0.20 \pm 0.20 \AA$ \\
$\mathrm{H} \delta_{\mathrm{F}}$ & $0.25 \pm 0.06$ & $-0.01 \pm 0.06 \AA$ & $0.25 \pm 0.06$ & $-0.01 \pm 0.07 \AA$ & $0.33 \pm 0.08$ & $0.00 \pm 0.07 \AA$ \\
$\mathrm{CN}_{1}$ & $2.33 \pm 0.37$ & $0.00 \pm 0.01 \mathrm{mag}$ & $1.02 \pm 0.31$ & $-0.01 \pm 0.01 \mathrm{mag}$ & $0.83 \pm 0.28$ & $0.00 \pm 0.02 \mathrm{mag}$ \\
$\mathrm{CN}_{2}$ & $2.84 \pm 0.40$ & $0.00 \pm 0.01 \mathrm{mag}$ & $0.84 \pm 0.26$ & $-0.01 \pm 0.01 \mathrm{mag}$ & $0.83 \pm 0.26$ & $0.00 \pm 0.01 \mathrm{mag}$ \\
$\mathrm{Ca} 4227$ & $0.19 \pm 0.30$ & $0.68 \pm 0.30$ & $0.17 \pm 0.32$ & $0.70 \pm 0.33$ & $0.03 \pm 0.24$ & $0.84 \pm 0.25$ \\
$\mathrm{H} \gamma_{\mathrm{A}}$ & $1.03 \pm 0.20$ & $-0.07 \pm 0.09 \AA$ & $0.35 \pm 0.05$ & $-0.01 \pm 0.08 \AA$ & $0.54 \pm 0.09$ & $-0.06 \pm 0.08 \AA$ \\
$\mathrm{H} \gamma_{\mathrm{F}}$ & $0.82 \pm 0.22$ & $0.02 \pm 0.06 \AA$ & $0.39 \pm 0.12$ & $0.03 \pm 0.06 \AA$ & $0.53 \pm 0.15$ & $0.03 \pm 0.06 \AA$ \\
$\mathrm{Fe} 4383$ & $1.85 \pm 0.29$ & $-0.32 \pm 0.15 \AA$ & $0.84 \pm 0.13$ & $-0.31 \pm 0.15 \AA$ & $1.39 \pm 0.20$ & $-0.33 \pm 0.14 \AA$ \\
$\mathrm{Ca} 4455$ & $0.94 \pm 0.29$ & $0.07 \pm 0.29$ & $1.07 \pm 0.31$ & $-0.10 \pm 0.31$ & $0.92 \pm 0.42$ & $0.10 \pm 0.42$ \\
$\mathrm{C}_{2} 4668$ & $1.05 \pm 0.16$ & $0.01 \pm 0.17$ & $0.85 \pm 0.13$ & $0.20 \pm 0.13$ & $0.80 \pm 0.09$ & $0.26 \pm 0.10$ \\
$\mathrm{H} \beta$ & $1.73 \pm 0.76$ & $0.13 \pm 0.06 \AA$ & $-2.87 \pm 1.14$ & $0.13 \pm 0.06 \AA$ & $2.13 \pm 0.86$ & $0.14 \pm 0.06 \AA$ \\
$\mathrm{Mg} g_{\mathrm{b}}$ & $0.63 \pm 0.20$ & $0.23 \pm 0.20$ & $0.60 \pm 0.24$ & $0.27 \pm 0.24$ & $0.39 \pm 0.19$ & $0.47 \pm 0.19$ \\
$\mathrm{Fe} 5335$ & $0.87 \pm 0.15$ & $0.07 \pm 0.15$ & $0.91 \pm 0.15$ & $0.04 \pm 0.16$ & $0.95 \pm 0.15$ & $0.00 \pm 0.15$ \\
\hline
\end{tabular}

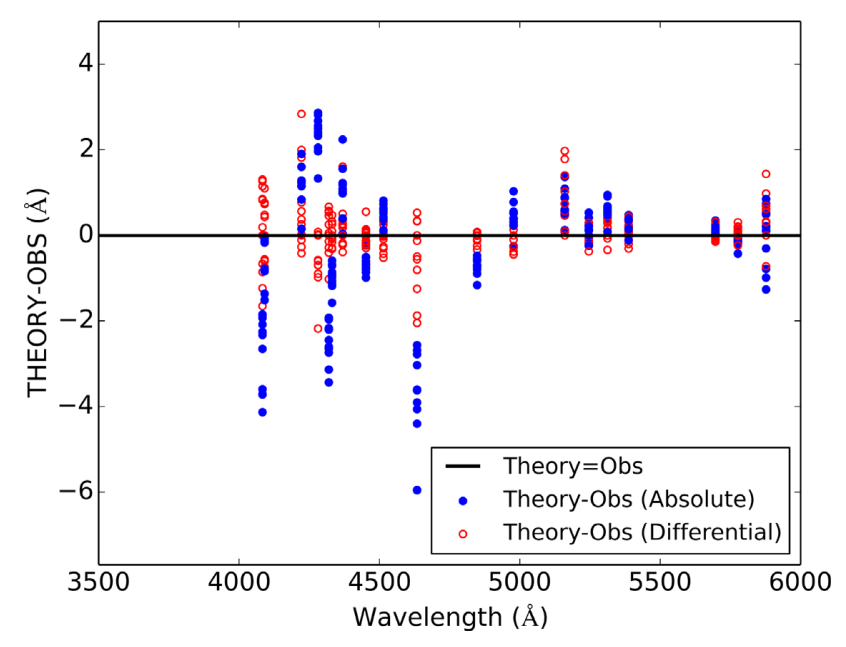

Figure 11. Comparison between the differential and absolute predictions of line strengths for the 19 indices, with units of $\AA$, as a function of wavelength. This is illustrated for the CAP Cool Giant models, with the same parameter cuts as Table 5, leaving 11 stars. The vertical axis shows differences between theoretical and observed index values. Red and blue points represent the differential and absolute application of the models, respectively. The absolute models have been produced with parameters that match those of Cenarro et al. (2007) MILES parameters.

models was corrupted. This corruption does not affect any of this work, but is discussed and illustrated in Appendix A.

\subsection{Model strengths and weaknesses}

Comparisons between models were discussed in Sections 4 and 5. Here, we summarize the main strengths and weaknesses of each individual model compared to observations, in terms of absolute and differential behaviours.

We find that all three models do not fit the Balmer features well, in an absolute and differential analysis, with the greatest problems seen in cool stars models in an absolute sense (Fig. 1) and in all star-types in a differential sense (Fig. 6). All three models do quite well at predicting iron-sensitive features (Figs 2 and 7). All models tend to underpredict $\mathrm{C}_{2} 4668$ line strengths in an absolute comparsion (Fig. 4). Calcium- and sodium (Figs 5 and 10)-sensitive features also show
Table 7. RMS scatter about the Theory $=$ Obs line of the three different applications of CAP Cool Giant model predictions. The columns represent the index name, the differential predictions, absolute predictions, and differential predictions fixing the $\alpha$ response to zero, respectively. In general the differential scatter is smaller or performing the same as the absolute behaviour.

\begin{tabular}{lccc}
\hline Index & $\begin{array}{c}\text { Absolute } \\
\AA\end{array}$ & $\begin{array}{c}\text { Differential } \\
\AA\end{array}$ & $\begin{array}{c}\text { Differential } \\
(\alpha \text {-fixed }) \AA\end{array}$ \\
\hline $\mathrm{H} \delta_{\mathrm{A}}$ & 2.86 & 1.02 & 0.59 \\
$\mathrm{H} \delta_{\mathrm{F}}$ & 0.75 & 0.60 & 0.36 \\
$\mathrm{Ca} 4227$ & 1.14 & 1.23 & 1.10 \\
$\mathrm{G} 4300$ & 2.39 & 0.86 & 0.51 \\
$\mathrm{H} \gamma_{\mathrm{A}}$ & 2.54 & 0.49 & 0.51 \\
$\mathrm{H} \gamma_{\mathrm{F}}$ & 1.00 & 0.26 & 0.23 \\
$\mathrm{Fe} 4383$ & 1.13 & 0.56 & 0.73 \\
$\mathrm{Ca} 4455$ & 0.69 & 0.22 & 0.18 \\
$\mathrm{Fe} 4531$ & 0.53 & 0.28 & 0.29 \\
$\mathrm{C}_{2} 4668$ & 4.03 & 1.00 & 1.01 \\
$\mathrm{H} \beta$ & 0.74 & 0.22 & 0.22 \\
$\mathrm{Fe} 5015$ & 0.53 & 0.30 & 0.27 \\
$\mathrm{Mg}$ & 0.77 & 1.03 & 0.86 \\
$\mathrm{Fe} 5270$ & 1.08 & 1.02 & 1.07 \\
$\mathrm{Fe} 5335$ & 0.69 & 0.38 & 0.43 \\
$\mathrm{Fe} 5406$ & 0.36 & 0.36 & 0.34 \\
$\mathrm{Fe} 5709$ & 0.50 & 0.54 & 0.54 \\
$\mathrm{Fe} 5782$ & 0.34 & 0.27 & 0.24 \\
$\mathrm{Na}$ & 0.59 & 0.73 & 0.72 \\
\hline
\end{tabular}

fairly good agreement with the data, with no clear systematics in both an absolute and differential sense, other than those noted below.

\subsubsection{Conroy}

Recall that in the absolute comparsions (Section 4), Conroy models were produced via interpolation in a pre-existing grid. Some systematic offsets between Conroy models and observations are seen in the absolute comparisons of magnesium-sensitive features, with the cool star models overpredicting feature strengths (Fig. 3). Conroy Cool Giant and Turn-off models tend to overpredict magnesiumsensitive line strengths but show a good fit for Cool Dwarf stars, in the differential analysis (Fig. 8). For $\mathrm{CN}_{1}$ and $\mathrm{CN}_{2}$ indices, Conroy 

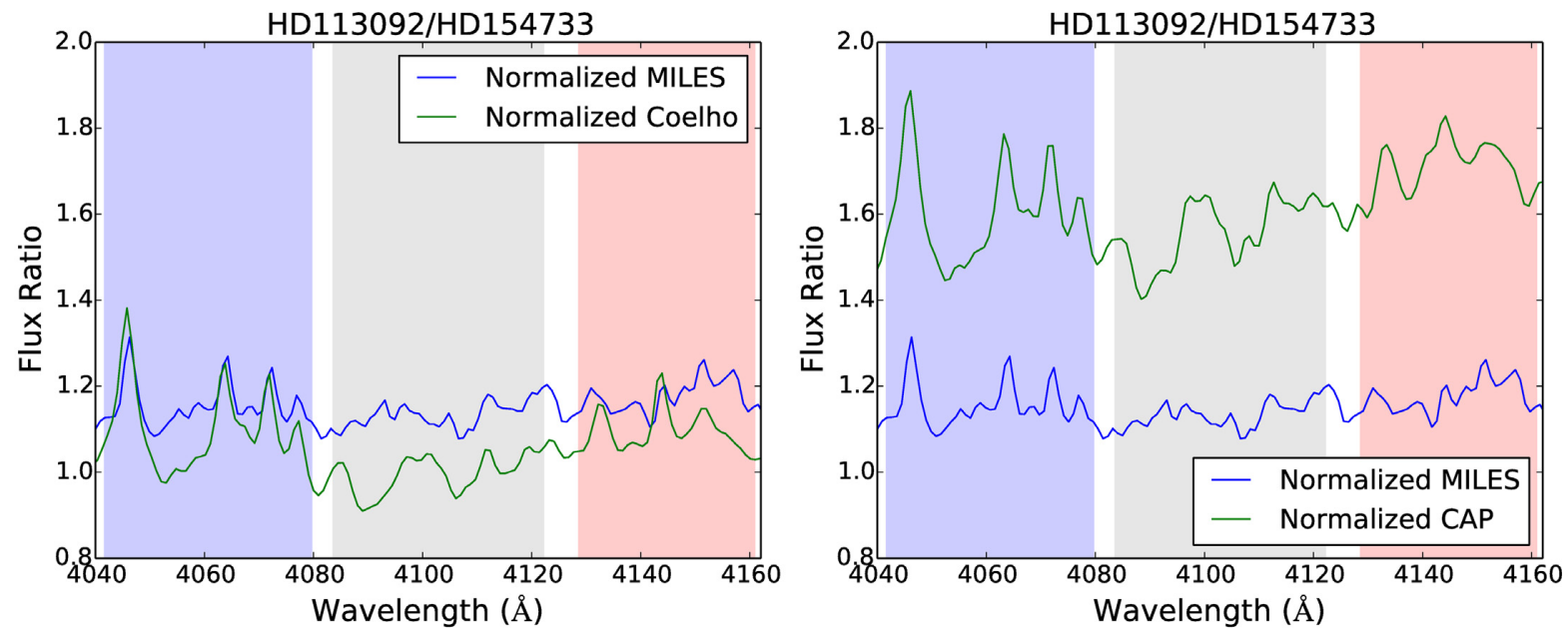

Figure 12. Spectral ratios in $C_{2} 4668$ region. The green and blue lines show a normalized (HD113092/HD154733) spectrum for the Cool Giant models and equivalent MILES stars, respectively. The left- and right-hand panels show the Coelho and CAP models, respectively. The red vertical lines show the positions of a few Swan bands. The blue, red, and grey areas represent the blue continuum, red continuum, and index band of the $\mathrm{C}_{2} 4668$ Lick index definition, respectively.
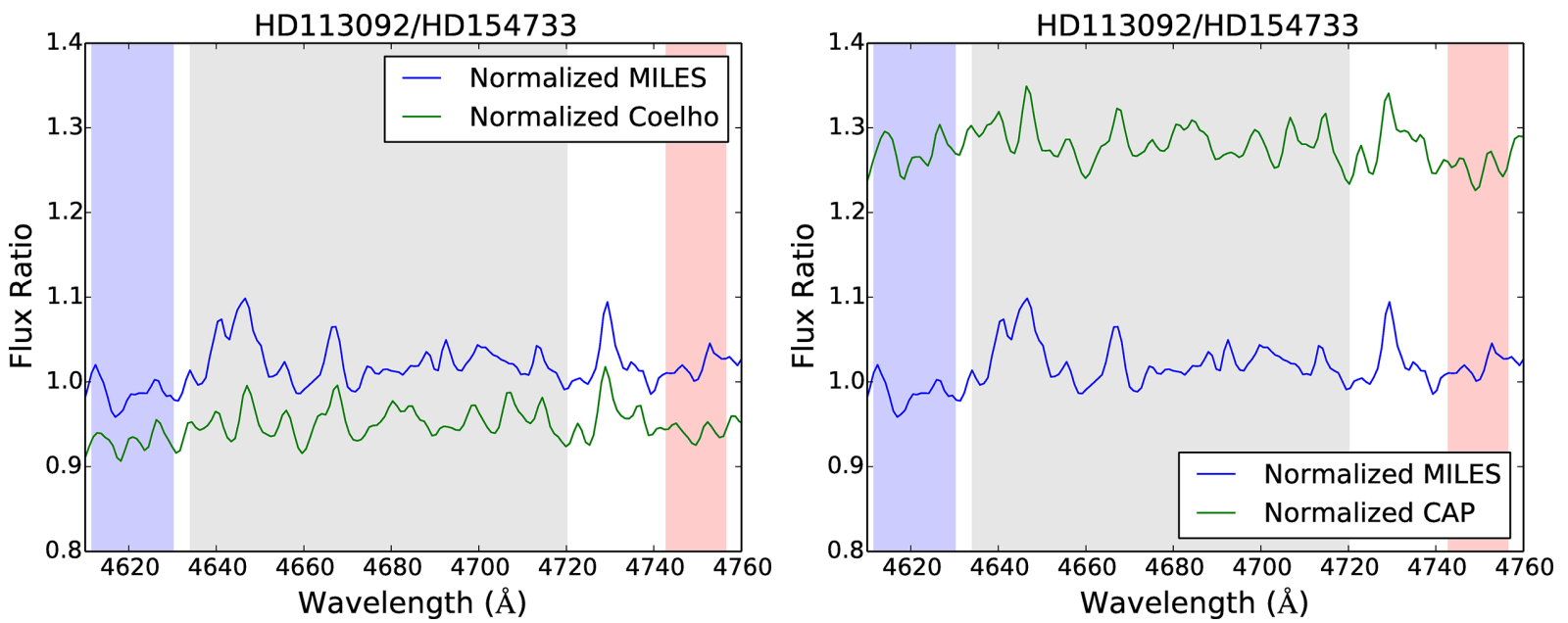

Figure 13. Spectral ratios in $\mathrm{H} \delta_{\mathrm{A}}$ region. The green and blue lines show a normalized (HD113092/HD154733) spectrum for the Cool Giant models and equivalent MILES stars, respectively. The left- and right-hand panels show the Coelho and CAP models, respectively. The blue, red, and grey areas represent the blue continuum, red continuum, and index band of the $\mathrm{H} \delta_{\mathrm{A}}$ Lick index definition, respectively.

cool star models overpredict line strengths in an absolute sense (Fig. 4). In the differential case, Conroy cool star models show a smaller range than the data (Fig. 9). Metal-poor Turn-off stars are underpredicted in $\mathrm{CN}_{2}$ for Conroy models, in a differential analysis (Fig. 9). Problems with Cool Dwarf models are found for $\mathrm{C}_{2} 4668$ in the differential analysis, with problems found in the base star that results in some outliers (Fig. 9). Ca4455 is overpredicted for all star types in an absolute sense (Fig. 5).

\subsubsection{Coelho}

Coelho models slightly overpredict cool star line strengths in magnesium-sensitive features for the absolute comparsion (Fig. 3) with overpredictions seen in the Cool Giant and Turn-off stars for the differential analysis (Fig. 8). Coelho Cool Dwarf line strengths are fit well for the magnesium-sensitive indices in the differential analysis. $\mathrm{CN}$ and $\mathrm{C}_{2} 4668$ indices are fit well in the differential analysis for all star types (Fig. 9). Coelho models also do well at fitting the $\mathrm{CN}$ indices in the absolute comparisons (Fig. 4).

\subsubsection{CAP}

CAP models generally show good agreement in magnesiumsensitive features, for the absolute comparsion (Fig. 3), with very slight overpredictions seen in the Cool Giant stars. Overpredictions are seen for the Cool Giant and Turn-off stars for the differential analysis (Fig. 8). CAP Cool Dwarf line strengths are fit well for the magnesium-sensitive indices in the differential analysis. $\mathrm{CN}$ and $\mathrm{C}_{2} 4668$ indices are fit well in the differential analysis for all star types (Fig. 9). However, they overpredict cool star $\mathrm{CN}$ line strengths in the absolute comparisons (Fig. 4). 


\section{CONCLUSIONS}

In this study, we have tested both the differential and absolute line-strength predictions of three state-of-the-art theoretical stellar model libraries, using empirical MILES stellar spectra. First, we directly tested three stellar model libraries, matching the parameters of $T_{\text {eff }}, \log g,[\mathrm{Fe} / \mathrm{H}]$, and $[\alpha / \mathrm{Fe}]$ of MILES stars to study trends and aid the differential tests. We then used response functions to account for changes in $[\mathrm{Fe} / \mathrm{H}]$ and $[\alpha / \mathrm{Fe}]$ abundances. The latest response functions calculated here are made publicly available in supplementary data online.

In summary, we find that:

(i) Differences between models are generally less significant than the ways in which models vary from the data.

(ii) All three models libraries do well at predicting abundance pattern effects in certain features, particularly those sensitive to iron and sodium.

(iii) Problems exist in the Balmer features of all models, with overprediction of the variation in $\mathrm{H} \delta$ and $\mathrm{H} \gamma$ indices present in cool star models. There is no clear abudance pattern correlations shown in $\mathrm{H} \beta$, however there is a weak abundance effect with $\mathrm{H} \beta_{0}$ (see Appendix B).

(iv) Using diagnostic index and spectral plots (like those shown in Figs 9 and 12), we identified a corrupted file of the transition D-A of $C_{2}$, describing the Swan bands in Coelho (2014) models. This corrupted file was corrected for this work. Appendix A (Figs A1 and A2) shows the effects of that corruption and its correction.

(v) As expected, the absolute differences between models and observations are generally worse than using the differential behaviour. This was investigated using the CAP Cool Giant models. In the application of these models, the differential approach produces less or similar scatter about the agreements with observations than the absolute predictions. In particular, the differential predictions of some hydrogen features are scattered by a factor of $\sim 2$ less than the absolute predictions. A large reduction in scatter between the two approaches is also seen in G4300 and $\mathrm{C}_{2} 4668$ indices.

(vi) In general, the largest differences between the observations and absolute model predictions are seen at lower temperatures, which may be explained by the omission of non-LTE and 3D geometry effects in all the models. The features that are largely affected in this temperature regime are the higher order Balmer features, with models overpredicting variations of line-strength index with abundance pattern in both differential and absolute predictons.

This work highlights the benefits of a differential approach to modelling abundance patterns. However, it still has its limitations and errors that we have attempted to show in this paper. Two caveats of this analysis have been highlighted in Section 6. We have not investigated if different stellar parameters for the MILES stars would affect our conclusions. We have also not attempted to study any abundances differences other than $[\alpha / \mathrm{Fe}]$, such as $\mathrm{C}, \mathrm{N}$, and $\mathrm{O}$, which might affect the empirical stars but are not changed from scaled solar in the models. If more abundances are measured for MILES stars, these effects can be studied in future. However, it can be seen that different models produce slightly different predictions of abundance pattern effects, and awareness of this will be important in the application of these models. We have shown that using the models' differential predictions of abundance pattern effects produces a better agreement with observations than using the absolute predictions, particularly at bluer wavelengths. This finding will be used in the generation of a new semi-empirical model library of stars that will make use of both observed spectra and differential predictions of theoretical spectra.

\section{ACKNOWLEDGEMENTS}

The authors would like to thank the STFC for providing ATK with the studentship for his PhD studies as well as the IAC for providing the support and funds that allowed ATK to visit the institute on two occasions. AES acknowledges travel support from grant AYA2016-77237-C3-1-P from the Spanish Ministry of Economy and Competitiveness (MINECO). PYTHON code used for blurring spectra was written by Jesús Falcón-Barroso. The authors would like to thank S. Earp and the anonymous referee, whose comments and suggestions improved the quality of this manuscript.

\section{REFERENCES}

Allende Prieto C. et al., 2014, A\&A, 568, A7

Allende Prieto C., Koesterke L., Hubeny I., Bautista M. A., Barklem P. S., Nahar S. N., 2018, A\&A, 618, A25

Asplund M., Grevesse N., Sauval A. J., 2005, in Barnes T. G., III, Bash F. N., eds, ASP Conf. Ser. Vol. 336, Cosmic Abundances as Records of Stellar Evolution and Nucleosynthesis. Astron. Soc. Pac., San Francisco, p. 25

Asplund M., Grevesse N., Sauval A. J., Scott P., 2009, ARA\&A, 47, 481

Bell R. A., Paltoglou G., Tripicco M. J., 1994, MNRAS, 268, 771

Bembenek Z., Kepa R., Rytel M., 1997, JMS, 183, 1

Bertone E., Buzzoni A., Chávez M., Rodríguez-Merino L. H., 2008, A\&A, 485,823

Brooke J. S. A., Bernath P. F., Schmidt T. W., Bacskay G. B., 2013, J. Quant. Spec. Radiat. Transf., 124, 11

Brooke J. S. A., Ram R. S., Western C. M., Li G., Schwenke D. W., Bernath P. F., 2014, ApJS, 210, 23

Castelli F., 2005a, Mem. Soc. Astron. Ital. Suppl., 8, 25

Castelli F., 2005b, Mem. Soc. Astron. Ital. Suppl., 8, 34

Cenarro A. J. et al., 2007, MNRAS, 374, 664

Cervantes J. L., Vazdekis A., 2009, MNRAS, 392, 691

Coelho P., Barbuy B., Meléndez J., Schiavon R. P., Castilho B. V., 2005, A\&A, 443, 735

Coelho P., Bruzual G., Charlot S., Weiss A., Barbuy B., Ferguson J. W., 2007, MNRAS, 382, 498

Coelho P. R. T., 2014, MNRAS, 440, 1027

Colin R., Bernath P. F., 2010, JMS, 263, 120

Conroy C., van Dokkum P., 2012, ApJ, 747, 69

Conroy C., Graves G. J., van Dokkum P. G., 2014, ApJ, 780, 33

da Silva R., Milone A. C., Reddy B. E., 2011, A\&A, 526, A71

Delgado Mena E., Israelian G., González Hernández J. I., Bond J. C., Santos N. C., Udry S., Mayor M., 2010, ApJ, 725, 2349

Falcón-Barroso J., Sánchez-Blázquez P., Vazdekis A., Ricciardelli E., Cardiel N., Cenarro A. J., Gorgas J., Peletier R. F., 2011, A\&A, 532, A95

Gonneau A. et al., 2016, A\&A, 589, A36

Grevesse N., Sauval A. J., 1998, Space Sci. Rev., 85, 161

Gustafsson B., Edvardsson B., Eriksson K., Jørgensen U. G., Nordlund Å., Plez B., 2008, A\&A, 486, 951

Holtzman J. A. et al., 2015, AJ, 150, 148

Johnson C. I., Rich R. M., Kobayashi C., Kunder A., Koch A., 2014, AJ, 148,67

Kepa R., Para A., Rytel M., Zachwieja M., 1996, JMS, 178, 189

Koesterke L., 2009, in Hubeny I., Stone J. M., MacGregor K., Werner K., eds, AIP Conf. Ser. Vol. 1171, Am. Inst. Phys., New York, Quantitative Spectroscopy in 3D, p. 73

Korn A. J., Maraston C., Thomas D., 2005, A\&A, 438, 685

Kurucz R., 1993, ATLAS9 Stellar Atmosphere Programs and 2 km/s grid. Kurucz CD-ROM No. 13, Smithsonian Astrophysical Observatory, Cambridge, MA

Kurucz R. L., 1979, ApJS, 40, 1 
Kurucz R. L., 1996, in Strassmeier K. G., Linsky J. L., eds, Proc. IAU Symp. Vol. 176, Stellar Surface Structure, Kluwer Academic Publishers, Dordrecht, p. 523

Kurucz R. L., 2005, Mem. Soc. Astron. Ital. Suppl., 8, 14

Kurucz R. L., , Avrett E. H., 1981, SAO Spec. Rep., 391, 1

Lee H.-c., et al., 2009, ApJ, 694, 902

Letarte B., Hill V., Tolstoy E., 2007, in Emsellem E., Wozniak H., Massacrier G., Gonzalez J.-F., Devriendt J., Champavert N., eds, EAS Publ. Ser. Vol. 24, EAS Publications Series, EDP Sciences, Le Ulis, France, p. 33

Mannucci F., 2008, Chin. J. Astron. Astrophys. Suppl., 8, 143

Maoz D., Sharon K., Gal-Yam A., 2010, ApJ, 722, 1879

Martins L. P., Coelho P., 2007, MNRAS, 381, 1329

Mészáros S. et al., 2012, AJ, 144, 120

Milone A. D. C., Sansom A. E., Sánchez-Blázquez P., 2011, MNRAS, 414, 1227

Munari U., Sordo R., Castelli F., Zwitter T., 2005, A\&A, 442, 1127

Onodera M. et al., 2015, ApJ, 808, 161

Pagel B. E. J., 2009, Nucleosynthesis and Chemical Evolution of Galaxies, Cambridge University Press, Cambridge

Percival S. M., Salaris M., Cassisi S., Pietrinferni A., 2009, ApJ, 690, 427

Poggianti B. M., Barbaro G., 1997, A\&A, 325, 1025

Proctor R. N., Sansom A. E., 2002, MNRAS, 333, 517

Prugniel P., Koleva M., Ocvirk P., Le Borgne D., Soubiran C., 2007, in Vazdekis A., Peletier R., eds, Proc. IAU Symp. 241, Stellar Populations as Building Blocks of Galaxies, Kluwer Academic Publishers, Dordrecht, p. 68

Rose J. A., 1984, AJ, 89, 1238

Sánchez-Blázquez P. et al., 2006, MNRAS, 371, 703

Sansom A. E., Milone A. d. C., Vazdekis A., Sánchez-Blázquez P., 2013, MNRAS, 435, 952

Sbordone L., Bonifacio P., Castelli F., Kurucz R. L., 2004, Mem. Societa Astron. Ital. Suppl., 5, 93

Schiavon R. P., 2007, ApJS, 171, 146

Sesto L. A., Faifer F. R., Smith Castelli A. V., Forte J. C., Escudero C. G., 2018, MNRAS, 479, 478

Sharma K., Prugniel P., Singh H. P., 2016, A\&A, 585, A64

Sullivan M. et al., 2006, ApJ, 648, 868

Swan W., 1875, Trans. R. Soc. Edinburugh, 21, 411

Thomas D., Maraston C., Bender R., 2003, MNRAS, 339, 897

Thomas D., Maraston C., Bender R., Mendes de Oliveira C., 2005, ApJ, 621,673

Thomas D., Johansson J., Maraston C., 2011, MNRAS, 412, 2199

Trager S. C., Faber S. M., Worthey G., González J. J., 2000, AJ, 119, 1645

Tripicco M. J., Bell R. A., 1995, AJ, 110, 3035

Vazdekis A. et al., 2015, MNRAS, 449, 1177

Vazdekis A., 2011, LECTOR: Line-strengths in One-dimensional ASCII Spectra, Astrophysics Source Code Library, record ascl:1104.006

Vazdekis A., Sánchez-Blázquez P., Falcón-Barroso J., Cenarro A. J., Beasley M. A., Cardiel N., Gorgas J., Peletier R. F., 2010, MNRAS, 404, 1639

Villaume A., Brodie J., Conroy C., Romanowsky A. J., van Dokkum P., 2017, ApJ, 850, L14
Walcher C. J., Coelho P., Gallazzi A., Charlot S., 2009, MNRAS, 398, L44 Worthey G., 1994, ApJS, 95, 107

Worthey G., Ottaviani D. L., 1997, ApJS, 111, 377

Worthey G., Faber S. M., Gonzalez J. J., 1992, ApJ, 398, 69

Zachwieja M., 1995, J. Mol. Spectrosc., 170, 285

Zachwieja M., 1997, J. Mol. Spectrosc., 182, 18

\section{SUPPORTING INFORMATION}

Supplementary data are available at MNRAS online.

Please note: Oxford University Press is not responsible for the content or functionality of any supporting materials supplied by the authors. Any queries (other than missing material) should be directed to the corresponding author for the article.

\section{APPENDIX A: COELHO LATEST REVISIONS}

During the work for this paper, we identified a problem in the $\mathrm{C}_{2} 4668$ region in the Coelho models. The origin of this problem was found to be in the $\mathrm{C}_{2}$ line list of these models. Specifically there was a corruption with the file containing the D-A transition. This is the likely origin of the missing opacity, which can be seen around 4000 $\AA$ in the second panel in fig. 10 of Coelho (2014). This corruption led to the problems shown in Figs A1 and A2. It can be seen in the left-hand panel of Fig. A1 that there was an issue regarding a lack of variation in the normalized $\mathrm{C}_{2} 4668$ index for the corrupted (old) Coelho cool star models. This is clarified in the left-hand panel of Fig. A2, which shows that the corrupted Coelho models had almost no variation in the $\mathrm{C}_{2} 4668$ spectral region with an increase of 0.3 dex in carbon. The models were recomputed with a corrected $\mathrm{C}_{2}$ D-A transition for this work and we plot the corrected models in the right-hand panel of Fig. A1 and in both panels of Fig. A2 (green lines). It can be seen in the right-hand panel of Fig. A1 that the corruption is ammended and the models now lie in closer agreement with MILES observations. This improvement is reflected in the spectral plots in Fig. A2, with Coelho's new models showing strong absorption features of the Swan bands (Swan 1875; Gonneau et al. 2016). Typical features, orginating from the $(1,0)$ vibrational plus rotational transitions, in the Swan bands exist at 4684, 4697, 4715, and $4737 \AA$ and these locations are shown in Fig. A2. Numerically, this correction results in an increase in $\mathrm{C}_{2} 4668$ carbon response from 0.014 to $10.266 \AA$ and an increase in $\mathrm{C}_{2} 4668$ overall metal response from -0.042 to $1.552 \AA$ between the old and corrected Coelho models, respectively. The response functions presented in the online data incorporate these corrections for Coelho's models. These corrections will be present in future works involving Coelho models. This correction has negligible effect on the other indices. 

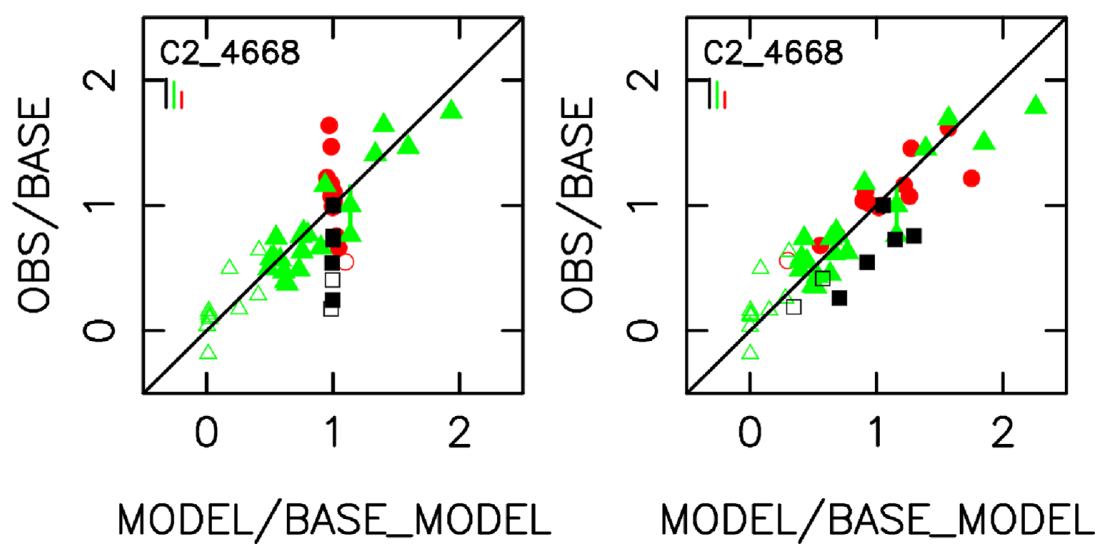

Figure A1. MILES Normalized Lick indices versus the Coelho (2014) models initially found in this study (Coelho Old) and the revised Coelho (Coelho New) models for $\mathrm{C}_{2} 4668$. Symbols and colours as in Fig. 6.
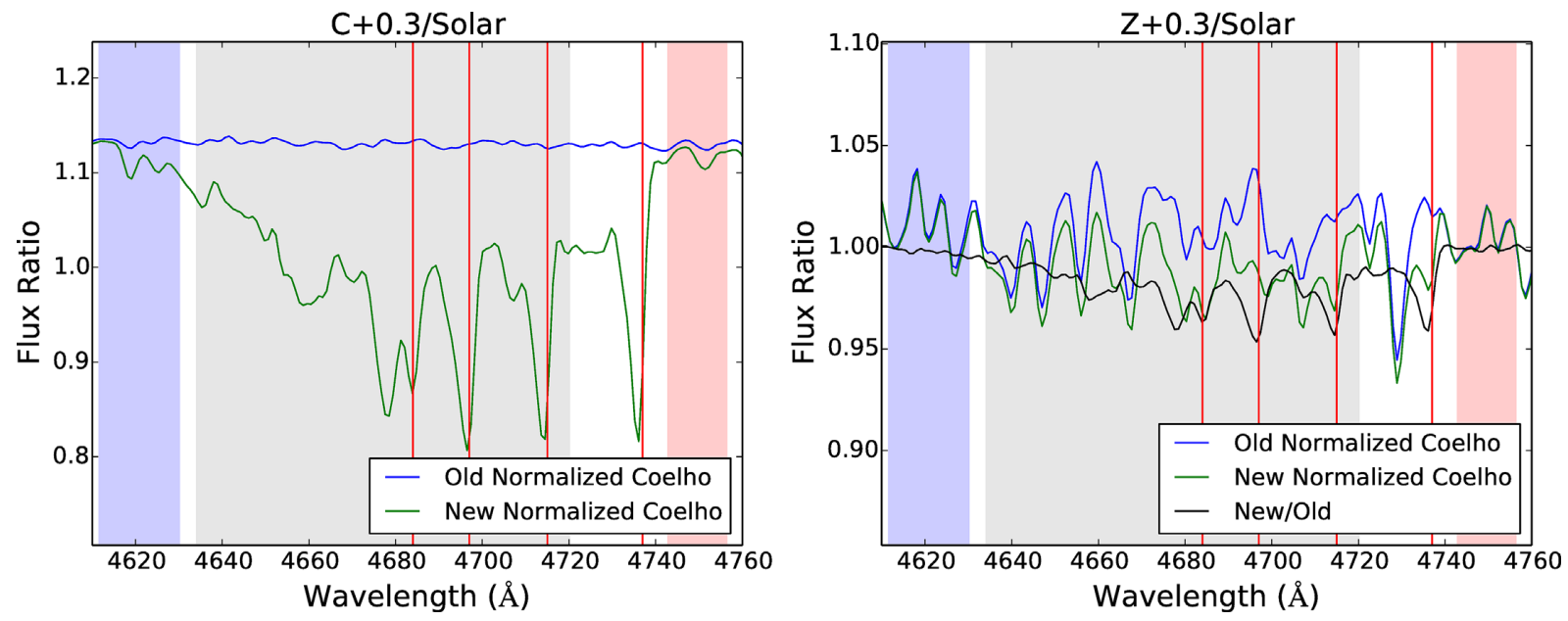

Figure A2. Spectral ratios in the $\mathrm{C}_{2} 4668$ region. The left-hand plot shows a comparison between normalized spectra (C+0.3/Solar) for the Coelho old and revised Cool Giant models. The right-hand plot shows a comparsion between normalized spectra $(\mathrm{Z}+0.3$ Solar $)$ for the Coelho old and revised Cool Giant models. In both plots the red vertical lines show positions of a few Swan Band $(1,0)$ features and the blue, red, and grey areas represent the blue continuum, red continuum, and index band of the $\mathrm{C}_{2} 4668$ Lick index definition, respectively. 


\section{APPENDIX B: H $\beta$ AND H $\beta_{0}$}

We explore how well models agree with observations for a variation of the standard Lick index for $\mathrm{H} \beta$. Cervantes \& Vazdekis (2009) defined $\mathrm{H} \beta_{0}$ with slightly different band limits than for $\mathrm{H} \beta$, in a search for better age representation. Fig. B1 shows the normalized index comparison for $\mathrm{H} \beta$ on the top row and $\mathrm{H} \beta_{0}$ on the bottom row, zoomed in to exclude six low $[\mathrm{Fe} / \mathrm{H}]$ Turn-off stars. In Table B1 we show the numerical differences in response functions of $\mathrm{H} \beta$ and $\mathrm{H} \beta_{0}$ indices for the three models tested in this study. Fig. B1 reveals a stronger correlation of $\mathrm{H} \beta_{0}$ with abundance pattern than found for $\mathrm{H} \beta$. This is highlighted in the larger $\mathrm{H} \beta_{0}$ responses to magnesium variations, seen in Table B1 (Column 7). Table B1 also shows that for all the models, $\mathrm{H} \beta_{0}$ has a stronger sensitivity to overall metallicity (Columns 9 and 15), compared to $\mathrm{H} \beta$, which is in agreement with the purely theoretical SSP models in Cervantes \& Vazdekis (2009). However, Cervantes \& Vazdekis (2009; their fig. 2) also found that in SSP models computed entirely from empirical MILES stars, $\mathrm{H} \beta_{0}$ is less sensitive to overall metallicity than $\mathrm{H} \beta$. Visual differences between the two definitions and further discussion can be found in Vazdekis et al. (2015).
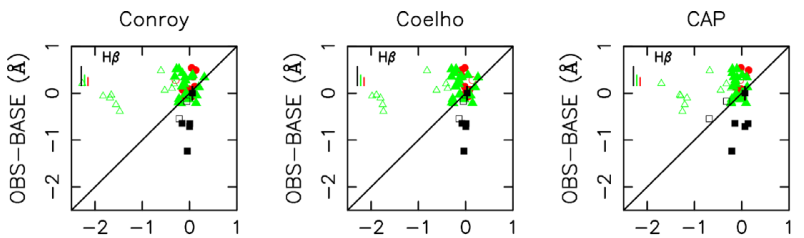

MODEL-BASE_MODEL
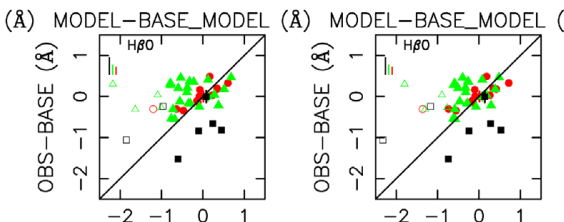

(§) MODEL-BASE_MODEL

(§) MODEL-BASE_MODEL

$(\AA)$

Figure B1. MILES Normalized Lick indices versus Conroy, Coelho, and CAP Model Normalized Lick indices derived from response functions, for $\mathrm{H} \beta$ and $\mathrm{H} \beta_{0}$ indices. The three star types are shown in each case, with green triangles, black squares, and red circles representing Turn-off, Cool Dwarf, and Cool Giant stars, respectively, as in Fig. 1.

Table B1. Comparison between $\mathrm{H} \beta$ and $\mathrm{H} \beta_{0}$ response functions. Column 1 is the model, Column 2 is index, Column 3 is the units of the index, Column 4 is the model base star index strength, and Columns 5-14 are the variation of the index strength when the element at the top of the column is increased by 0.3 dex ( 0.15 dex for $C$ in Conroy models). The last column shows the variation of index strength when there is an overall metallicity increase of 0.3 dex. Blank columns show that the element was not varied by the modeller.

\begin{tabular}{|c|c|c|c|c|c|c|c|c|c|c|c|c|c|c|}
\hline Model & Index & Units & $\mathrm{I}_{0}$ & $\mathrm{C}$ & $\mathrm{N}$ & $\mathrm{O}$ & $\mathrm{Mg}$ & $\mathrm{Fe}$ & $\mathrm{Ca}$ & $\mathrm{Na}$ & $\mathrm{Si}$ & $\mathrm{Cr}$ & $\mathrm{Ti}$ & {$[\mathrm{M} / \mathrm{H}]$} \\
\hline $\begin{array}{l}\text { Coelho Cool } \\
\text { Dwarf }\end{array}$ & $\mathrm{H} \beta$ & $\AA$ & -0.21 & -0.135 & 0.004 & 0.024 & -0.176 & & -0.03 & & & & & -0.048 \\
\hline $\begin{array}{l}\text { Coelho Cool } \\
\text { Dwarf }\end{array}$ & $\mathrm{H} \beta_{0}$ & $\AA$ & 2.242 & 0.019 & 0.011 & -0.001 & -0.359 & & 0.050 & & & & & 0.356 \\
\hline Coelho Cool Giant & $\mathrm{H} \beta$ & $\AA$ & -0.018 & -0.187 & 0.014 & 0.083 & -0.074 & & -0.012 & & & & & -0.07 \\
\hline Coelho Cool Giant & $\mathrm{H} \beta_{0}$ & $\AA$ & 2.801 & -0.060 & 0.025 & 0.021 & -0.190 & & 0.016 & & & & & 0.438 \\
\hline Coelho Turn-Off & $\mathrm{H} \beta$ & $\AA$ & 3.768 & -0.001 & 0.005 & 0.003 & 0.025 & & 0.003 & & & & & 0.258 \\
\hline Coelho Turn-Off & $\mathrm{H} \beta_{0}$ & $\AA$ & 5.054 & 0.007 & 0.002 & 0.003 & 0.050 & & 0.030 & & & & & 0.676 \\
\hline $\begin{array}{l}\text { Conroy Cool } \\
\text { Dwarf }\end{array}$ & $\mathrm{H} \beta$ & $\AA$ & -0.781 & -0.100 & 0.007 & & -0.494 & 0.304 & -0.021 & 0.046 & 0.046 & -0.080 & 0.057 & -0.116 \\
\hline $\begin{array}{l}\text { Conroy Cool } \\
\text { Dwarf }\end{array}$ & $\mathrm{H} \beta_{0}$ & $\AA$ & 1.435 & -0.101 & 0.013 & & -0.805 & 0.783 & 0.098 & 0.040 & 0.018 & 0.028 & 0.189 & 0.276 \\
\hline $\begin{array}{l}\text { Conroy Cool } \\
\text { Giant }\end{array}$ & $\mathrm{H} \beta$ & $\AA$ & 0.218 & -0.136 & 0.012 & & -0.171 & 0.313 & 0.007 & 0.007 & 0.052 & -0.056 & 0.043 & 0.091 \\
\hline $\begin{array}{l}\text { Conroy Cool } \\
\text { Giant }\end{array}$ & $\mathrm{H} \beta_{0}$ & $\AA$ & 3.222 & -0.128 & 0.032 & & -0.286 & 0.539 & 0.040 & 0.006 & 0.030 & 0.022 & 0.207 & 0.544 \\
\hline Conroy Turn-Off & $\mathrm{H} \beta$ & $\AA$ & 3.547 & -0.020 & 0.003 & & 0.032 & 0.098 & 0.007 & 0.001 & 0.040 & -0.030 & 0.081 & 0.253 \\
\hline Conroy Turn-Off & $\mathrm{H} \beta_{0}$ & $\AA$ & 4.853 & -0.030 & 0.001 & & 0.063 & 0.409 & 0.034 & 0.003 & 0.053 & 0.023 & 0.077 & 0.671 \\
\hline CAP Cool Dwarf & $\mathrm{H} \beta$ & $\AA$ & 0.147 & -0.427 & 0.000 & 0.170 & -0.508 & -0.111 & -0.057 & -0.021 & -0.049 & -0.114 & 0.108 & 0.019 \\
\hline CAP Cool Dwarf & $\mathrm{H} \beta_{0}$ & $\AA$ & 2.127 & -0.530 & 0.001 & 0.140 & -0.799 & 0.315 & -0.024 & -0.033 & -0.103 & -0.001 & 0.186 & 0.332 \\
\hline CAP Cool Giant & $\mathrm{H} \beta$ & $\AA$ & 0.449 & -0.457 & -0.001 & 0.336 & -0.290 & -0.071 & -0.024 & -0.009 & -0.060 & -0.093 & 0.187 & 0.117 \\
\hline CAP Cool Giant & $\mathrm{H} \beta_{0}$ & $\AA$ & 2.710 & -0.470 & 0.006 & 0.211 & -0.452 & 0.166 & -0.004 & -0.013 & -0.102 & -0.015 & 0.229 & 0.526 \\
\hline CAP Turn-Off & $\mathrm{H} \beta$ & $\AA$ & 3.864 & -0.034 & 0.001 & 0.013 & -0.099 & -0.182 & -0.014 & -0.004 & -0.070 & -0.044 & 0.072 & 0.157 \\
\hline CAP Turn-Off & $\mathrm{H} \beta_{0}$ & $\AA$ & 5.040 & -0.083 & 0.001 & 0.016 & -0.078 & 0.097 & -0.002 & -0.003 & -0.042 & -0.008 & 0.080 & 0.520 \\
\hline
\end{tabular}

This paper has been typeset from a $\mathrm{T}_{\mathrm{E}} \mathrm{X} / \mathrm{L} \mathrm{T}_{\mathrm{E}} \mathrm{X}$ file prepared by the author. 